University of Tennessee Health Science Center UTHSC Digital Commons

$12-2020$

\title{
Early Diagnosis of Alzheimer's Disease in the Primary Care Setting
}

Raymond R. Romano

University of Tennessee Health Science Center

Follow this and additional works at: https://dc.uthsc.edu/dissertations

Part of the Diagnosis Commons, Nervous System Diseases Commons, and the Other Nursing Commons

\section{Recommended Citation}

Romano, Raymond R. (https://orcid.org/0000-0003-1951-2412), "Early Diagnosis of Alzheimer's Disease in the Primary Care Setting" (2020). Theses and Dissertations (ETD). Paper 532. http://dx.doi.org/10.21007/ etd.cghs.2020.0567.

This Dissertation is brought to you for free and open access by the College of Graduate Health Sciences at UTHSC Digital Commons. It has been accepted for inclusion in Theses and Dissertations (ETD) by an authorized administrator of UTHSC Digital Commons. For more information, please contact jwelch30@uthsc.edu. 


\title{
Early Diagnosis of Alzheimer's Disease in the Primary Care Setting
}

\begin{abstract}
The burden of Alzheimer's disease (AD) affects not just the individual but also families, providers, and society. Early recognition and diagnosis of $A D$ may reduce cost by reducing interaction with the health care system, earlier initiation of treatment, and prolonging time to long- term care. Primary care providers, the first contact for diagnosis of patients with $A D$, are not fulfilling the potential of early diagnosis for a variety of reasons. Biomarkers of $A D$ emerge on average 15 to 20 years before clinical diagnosis, yet currently established biomarkers are not easily available in the primary care setting. A growing body of literature is focused on identifying additional non-invasive early signs of AD. The aims of this program of research were to understand factors contributing to the AD diagnosis variability in primary care settings and methods to improve early diagnosis by primary care providers. Four studies were undertaken to achieve these aims. The first study reported the results of an integrated review estimating the prevalence of missed diagnosis in primary care when compared to trained raters' diagnoses. The findings call to attention the difficulty primary care providers face to detect and diagnose $A D$ at all levels of the healthcare system. This led to the second study. Chronic pain is a common comorbid ailment seen in those with $A D$ and often is a driving factor of patients seeking medical care. In order to understand the pain experience in those with worsening cognition, the second study was a secondary analysis of a crosssectional age- and sex-matched two group cohort study and found that the experience of pain differs between males and females as a measure of cognition worsened suggesting a possible role of pain as a tool to distinguish those at risk for AD. This finding led to the third study, which was a narrative review conducted to describe how alterations in senses have been associated with the diagnosis of AD. The results suggested differences in smell, taste, vision, hearing, and proprioception were associated with different levels of the AD continuum but points out an obvious gap in the literature concerning other senses. This led to the fourth study examining evidence that the $\varepsilon 4$ allele of Apolipoprotein E modifies the experience of pain in those individuals carrying the allele such that greater temperatures are required to elicit pain and the experience of that pain is more unpleasant. Additional studies should expand on the results of this pilot study.
\end{abstract}

\section{Document Type}

Dissertation

Degree Name

Doctor of Philosophy $(\mathrm{PhD})$

Program

Nursing Science

Research Advisor

Michael A. Carter, DNSc

\section{Keywords}

Alzheimer's disease, Diagnosis, Pain, Primary Care

\section{Subject Categories}

Analytical, Diagnostic and Therapeutic Techniques and Equipment | Diagnosis | Diseases | Medicine and Health Sciences | Nervous System Diseases | Nursing | Other Nursing 


\section{University of TenNessee HeAlth Science Center}

DOCTORAL DisSERTATION

\section{Early Diagnosis of Alzheimer's Disease in the Primary Care Setting}

Author:

Raymond R. Romano
Advisor:

Michael A. Carter, DNSc

A Dissertation Presented for The Graduate Studies Council of The University of Tennessee Health Science Center in Partial Fulfillment of Requirements for the Doctor of Philosophy degree from The University of Tennessee

in

Nursing Science

College of Graduate Health Sciences

December 2020 
Chapter 2 @ 2020 by Wolters Kluwer Health, Inc.

Chapter 3 (C) 2020 by IOS Press.

Chapter 4 (C) 2020 by SAGE Publications.

All other material (C) 2020 by Raymond R. Romano.

All rights reserved.

Modified with permission Masters/Doctoral Thesis LaTeX Template

Version $2.5(8 / 27 / 2017)$

http://www.LaTeXTemplates . com

Creative Commons License CC BY-NC-SA 3.0 
To those who struggle with depression, anxiety, and shame. 


\section{Acknowledgements}

I would like to acknowledge my mentors, Drs. Michael Carter and Todd Monroe, without their patience, compassion, and guidance I would not be completing this work. I would like to thank Dr. Carolyn Graff whose leadership has been instrumental. I am grateful to Dr. Ronald Cowan whose expertise has elevated this work and Dr. Margaret Thorman Hartig whose experience has proven to be invaluable. I would also like to acknowledge Dr. Ann Aguanno who first exposed me to the world of research. I would like to acknowledge my family and friends, whose love and support helped guide and shape this dissertation. Lastly, I want to thank Jase, my fiance, who helped throughout by doing more laundry, smiling and nodding, and letting me be a bit more selfish during this process. 


\section{Abstract}

Raymond R. Romano

Early Diagnosis of Alzheimer's Disease in the

Primary Care Setting

The burden of Alzheimer's disease (AD) affects not just the individual but also families, providers, and society. Early recognition and diagnosis of AD may reduce cost by reducing interaction with the health care system, earlier initiation of treatment, and prolonging time to long- term care. Primary care providers, the first contact for diagnosis of patients with $\mathrm{AD}$, are not fulfilling the potential of early diagnosis for a variety of reasons. Biomarkers of AD emerge on average 15 to 20 years before clinical diagnosis, yet currently established biomarkers are not easily available in the primary care setting. A growing body of literature is focused on identifying additional non-invasive early signs of AD. The aims of this program of research were to understand factors contributing to the AD diagnosis variability in primary care settings and methods to improve early diagnosis by primary care providers. Four studies were undertaken to achieve these aims. The first study reported the results of an integrated review estimating the prevalence of missed diagnosis in primary care when compared to trained raters' diagnoses. The findings call to attention the difficulty primary care providers face to detect and diagnose AD at all levels of the healthcare system. This led to the second study. Chronic pain is a common comorbid ailment seen in those with $\mathrm{AD}$ and often is a driving factor of patients seeking medical care. In order to understand the pain experience in those with worsening cognition, the second study was a secondary analysis of a cross-sectional age- and sex-matched two group cohort study and found that the experience of pain differs between males and females as a measure of cognition worsened suggesting a possible role of pain as a tool to distinguish those at risk for AD. This finding led to the third study, which was a narrative review conducted to describe how alterations in senses have been associated with the diagnosis of AD. The results suggested differences in smell, taste, vision, hearing, and proprioception were associated with different levels of the AD continuum but points out an obvious gap in the literature concerning other senses. This led to the fourth study examining evidence that the $\varepsilon 4$ allele of Apolipoprotein E modifies the experience of pain in those individuals 
carrying the allele such that greater temperatures are required to elicit pain and the experience of that pain is more unpleasant. Additional studies should expand on the results of this pilot study. 


\section{Contents}

1 Introduction $\quad 1$

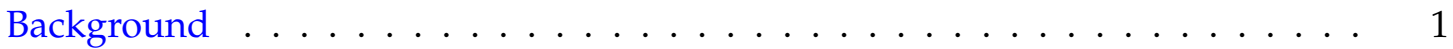

Theoretical Framework . . . . . . . . . . . . . . . . . . 2

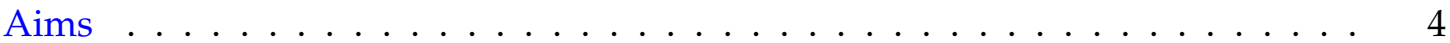

2 Factors Influencing Dementia Detection in Primary Care Setting 6

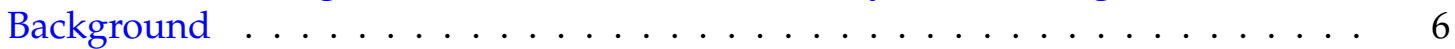

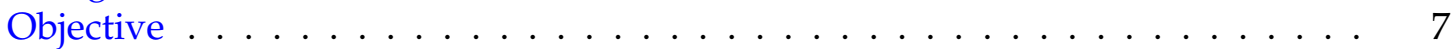

Methods ........................... 7



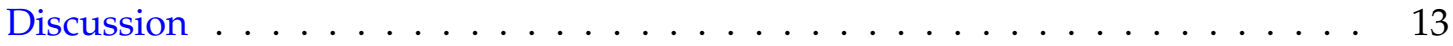

Gaps in Literature . . . . . . . . . . . . . . . . . . . . . 14

Limitations . . . . . . . . . . . . . . . . . . . . . . . . . 14

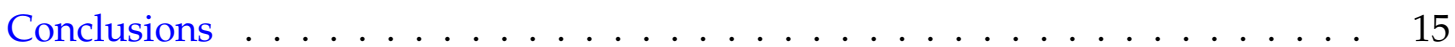

3 Cognitive Function with Perceptions of Pain: Sex Differences in Seniors 16

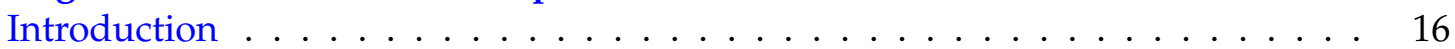

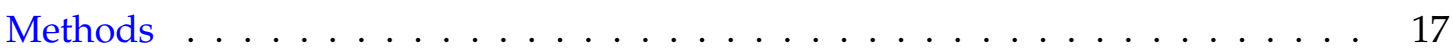

Study Design . . . . . . . . . . . . . . . . . . 17

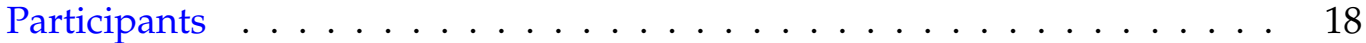

Screening and Enrollment . . . . . . . . . . . . . . . . . 18

Measures . . . . . . . . . . . . . . . . . . . . . . . 19

Thermal Stimulation Protocol $\ldots \ldots$. . . . . . . . . . . . . . . . . . . . . 19

Statistical Analysis . . . . . . . . . . . . . . . . . . . . . . . . 19

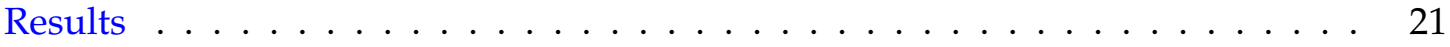

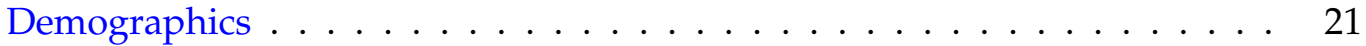

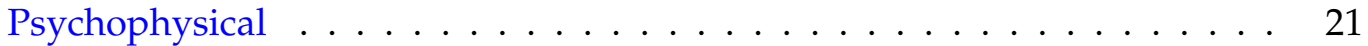

Associations of MMSE with Psychophysical Reports . . . . . . . . . . . . . . . 22

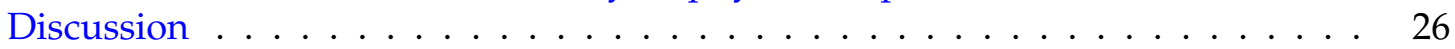

4 Sensory Changes as a Biomarker for Alzheimer's Disease 28

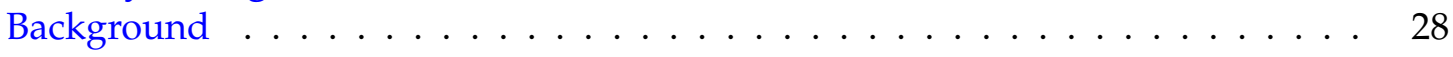

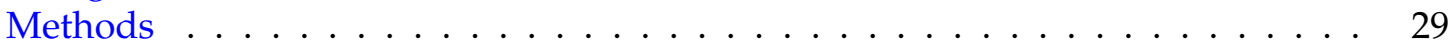




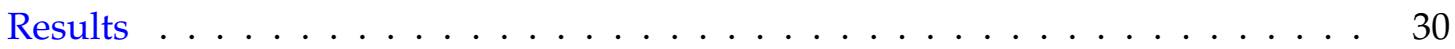

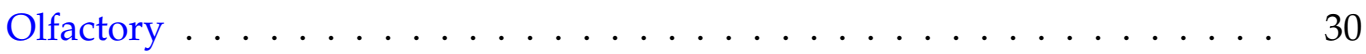





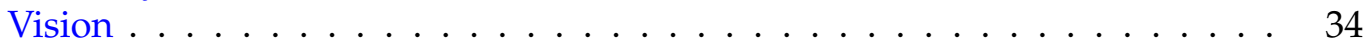

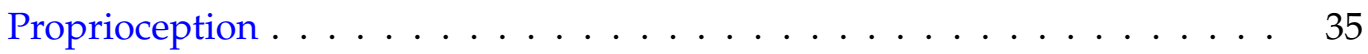

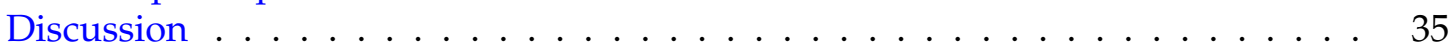

Implications for Research $\ldots \ldots \ldots \ldots \ldots$

Limitations . . . . . . . . . . . . . . . . . . . 36

5 Could an Altered Evoked Pain Response Be a Phenotypic Biomarker for Alzheimer's Disease Risk? $\quad 38$

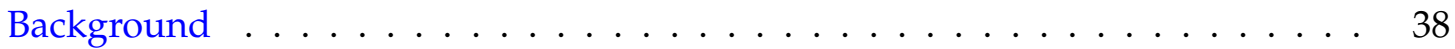

Methods ............................. 40

Study Participants . . . . . . . . . . . . . . . . . . . . . . . . . . . . . 40

Screening and Enrollment . . . . . . . . . . . . . . . . . . . . . 40

Thermal Stimulation Protocol (Psychophysics) . . . . . . . . . . . . . . . 40

APOE4 Status Determination . . . . . . . . . . . . . . . . . . . . . . 41

Statistical Analysis . . . . . . . . . . . . . . . . . . . 41

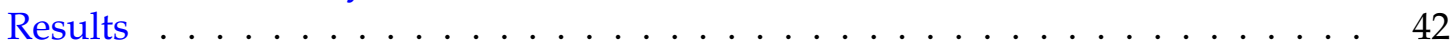



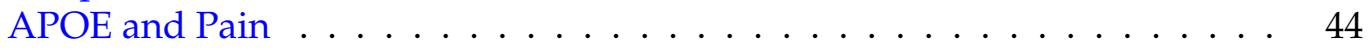

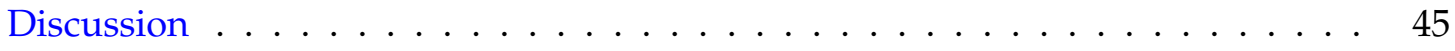

6 Discussion $\quad 47$

Summary . . . . . . . . . . . . . . . . . . . 47

Limitations . . . . . . . . . . . . . . . . . . . . 47

Recommendations for Future Directions . . . . . . . . . . . . . . . . . 48

List of References $\quad 49$

$\begin{array}{ll}\text { Vita } & 62\end{array}$ 


\section{List of Tables}

2.1 A descriptive comparison of studies. . . . . . . . . . . . . . . . . . . 11

2.2 Descriptive comparison of studies quality. . . . . . . . . . . . . . . 11

2.3 Descriptive comparison of accuracy of dementia diagnosis. . . . . . . . . . . 11

2.4 Factors identified contributing to detection and care of dementia . . . . . . . 12

3.1 Distribution of MMSE by gender groups. . . . . . . . . . . . . . . 22

3.2 Demographic and clinical characteristics of the participants. . . . . . . . 23

3.3 Psychophysics of temperature thresholds necessary to produce warmth, mild pain, or moderate pain and unpleasantness ratings at each condition. . . . . 24

3.4 Correlations of MMSE scores with temperature and unpleasantness ratings at each threshold level for the entire sample and within gender groups. . . . 25

4.1 Description of sample of 12 studies on olfaction as a biomarker. . . . . . . . 32

4.2 Quality scores of auditory, vision, proprioception, and taste studies. . . . . . 37

5.1 Demographic and sample characteristics. . . . . . . . . . . . . . . . 43

5.2 Experience of pain between APOE4 negative and positive participants. . . . 44 


\section{List of Figures}

2.1 Consort prisma diagram. . . . . . . . . . . . . . . . 9

3.1 Numerical descriptor scale used to measure affective unpleasantness and sensory intensity. . . . . . . . . . . . . . . . . 20

4.1 Consort prisma flow diagram. . . . . . . . . . . . . . . . . . . 31

5.1 Consort flow diagram. . . . . . . . . . . . . . . . . . . 42 


\title{
List of Abbreviations
}

\author{
A Beta Amyloid Deposition \\ AD Alzheimer's Disease \\ APOE Apolipoprotein E \\ BPI-SF Brief Pain Inventory Short Form \\ CDK5 Cyclin Dependent Kinase 5 \\ CNS Central Nervous System \\ CSF Cerebral Spinal Fluid \\ GDS-15 Geriatric Depression Scale-Short Form \\ IQR Inter-Quartile Range \\ IRB Institutional Review Board \\ MCI Mild Cognitive Impairment \\ MMSE Mini-Mental State Examination \\ $\mathrm{N} \quad$ Neurodegeneration \\ NHLBI National heart, Lung, and Blood Institute \\ NIA National Institute on Aging \\ NP Nurse Practitioners \\ PCP Primary Care Providers \\ SES Hollingshead Four-Factor Index of Socioeconomic Status \\ STAI Spielberger State-Trait Anxiety Inventory \\ T Pathological Tau \\ UBACC University of California San Diego Brief Assessment of Capacity to Consent
}




\section{Chapter 1}

\section{Introduction}

\section{Background}

An estimated financial benefit of $\$ 7$ trillion can be expected to the United States health care system if 70\% of those with Alzheimer's disease (AD) are diagnosed ("2020 Alzheimer's disease facts and figures" 2020). Since first described in 1907 by Alios Alzheimer, who noted the presence of abnormal fibrous inclusions within the cytoplasm of pyramidal neurons of a 50 year old age woman, the presence of these "hallmarks" of AD on autopsy remains the only definitive means of diagnosis of AD (Hippius and Neundorfer, 2003). Current estimates suggest that half of the 5.8 million Americans age 65 and older living with AD are undiagnosed ("2020 Alzheimer's disease facts and figures" 2020). Primary care providers (PCPs) have been identified as key to improve earlier recognition of $\mathrm{AD}$, though, there is evidence suggesting a lack of confidence in their own diagnostic skills and care management ("2020 Alzheimer's disease facts and figures" 2020).

In the primary care setting AD presents variably with subtle changes over the course of months to years and PCPs find it difficult to recognize these early symptoms in patients (Knopman, Donohue, and Gutterman, 2000; Martin, Kelly, et al., 2015). Many providers wrongly misattribute small changes in cognitive function as a process of normal aging (Knopman, Donohue, and Gutterman, 2000). Timeliness of diagnosis has been shown to vary from 18 to 30 months, and in some cases, up to four years (Dubois, Padovani, et al., 2016). There is evidence of variation in the ability of primary care providers to consistently detect and diagnose AD (Bradford et al., 2009). A lack of specialty services available is a common complaint amongst PCPs when surveyed on the difficulties of care for patients with AD (Bradford et al., 2009).

Interventions to improve diagnosis have seldom focused on overcoming the barriers identified in previous studies and the effects of interventions aimed at improving diagnosis are limited (Iliffe, J. Wilcock, et al., 2010; Koch and Iliffe, 2011). The results of empirical studies aimed to evaluate interventions to improve early diagnosis have been limited (Koch and Iliffe, 2011). Educational interventions, focusing on improving care through 
seminars, workshops, small-group learning, and decision support software found no evidence of improved diagnosis between intervention or control groups (Koch and Iliffe, 2011). The service based interventions of initiating dementia case-managers did not show increased rates of diagnosis (Koch and Iliffe, 2011). An in-home assessment by a specialist nurse only increased diagnosis by 20\% (Perry et al., 2008).

PCPs are advised to focus on the evaluation of the clinical consequences of AD, using clinical assessment and the results of neuropsychological testing, to diagnose AD. This does not incorporate the currently widely held understanding of AD which includes measures of biomarkers (Jack et al., 2018; Tolea and Galvin, 2013). As the majority of older adults with AD are diagnosed by their PCP, focus should be paid to understanding how primary care providers view the diagnosis of $\mathrm{AD}$ and what tools or resources can be made available to them to improve their diagnostic abilities ("2020 Alzheimer's disease facts and figures" 2020).

\section{Theoretical Framework}

The National Institute on Aging (NIA) developed a framework for the diagnosis of AD that has shifted idea of AD as a collection of clinical consequences and towards a biological process that incorporates measurable physiological parameters or biomarkers (Jack et al., 2018). Current biomarkers of AD are grouped into those of beta amyloid deposition (A), pathologic tau $(\mathrm{T})$, and neurodegeneration $(\mathrm{N})$ and are typically gathered by advanced imaging and lumbar puncture to obtain cerebrospinal fluid (Jack et al., 2018). The NIA framework describes how variation of biomarker profiles can be used to diagnose those with AD and highlights the alterations of biological processes occurring during a preclinical phase or before disease is recognized clinically. The earliest markers of AD which can be detected at least 10-20 years before the onset of clinical symptoms are the depositions of $\mathrm{A} \beta$ as measured with advance neuroimaging and within the cerebrospinal fluid (Long and Holtzman, 2019).

A $\beta$ peptides are produced by the sequential cleavage of $\beta$-amyloid precursor protein by $\beta$-secretase enzyme and $\gamma$-secretase into smaller units (Long and Holtzman, 2019). $\mathrm{A} \beta$ peptides are prone to aggregate to form oligomers, protofibrils, and fibrils. The $A \beta 42$ oligmer has a greater propensity to aggregated and can be found at the center of synthetic amyloid plaques (Long and Holtzman, 2019). There is evidence of $A \beta$ uptake by microglia and intracellualr seeding in a prion-like manner leads to aggregation of oligomers leading to the amyloid plaques visible on autopsy (Long and Holtzman, 2019). Although not proven, the deposition of $A \beta$ is thought to be the initiating steps of AD pathology supported by evidence that in genetic forms of AD there is an increase in $A \beta 42, A \beta$ production, and $\mathrm{A} \beta$ fribrillinogenic properties (Long and Holtzman, 2019). Additionally, the strongest genetic risk factor for $\mathrm{AD}$, apolipoprotien $\mathrm{E}$ (APOE) influences $\mathrm{A} \beta$ seeding and clearance 
(Long and Holtzman, 2019). The aggregation and deposition of $\mathrm{A} \beta$ is thought to cause down stream effects leading to AD (Jack et al., 2018; Long and Holtzman, 2019).

$\mathrm{A} \beta$ deposition may be required for the progression of tau pathology in $\mathrm{AD}$ as tau pathology does not progress without co-occurring amyloid pathology, and the rate of amyloid accumulations predicts onset of tau accumulation (Long and Holtzman, 2019). In AD tau propagates throughout the AD brain in a stereotyped fashion and can be classified using the Braak staging (Braak et al., 2003; Long and Holtzman, 2019). The tau protein is associated with microtubule assembly, stabilization of neuronal axons and microtubule transport (Long and Holtzman, 2019). Hyperphosporylated tau tends to aggregated and fibrilized. The pattern of tau misfolding in AD is different to other tau pathologies (Long and Holtzman, 2019). Tau is measured in cerebral spinal fluid (CSF) and with Tau PET imaging (Jack et al., 2018).

Tau pathology may lead to neurodegeneration (Jack et al., 2018). Brains of those with AD show cerebral cortical atrophy which does not involve the frontotemporal cortex and spares primary motor, sensory, and visual areas (Risacher and Saykin, 2013). AD pathology also leads to symmetrical dilation of the lateral ventricles with significant atrophy of the hippocampus and dilation of the adjacent temporal horn of the lateral ventricle (Risacher and Saykin, 2013). Biomarkers of neurodegeration have many causes and are not associated with neuropathological findings used to diagnose AD (Jack et al., 2018). Measures of neurodegeneration together with amyloid and tau measures are better at predicting cognitive decline than amyloid studies alone (Jack et al., 2018).

The cut point for each AT(N) biomarker describes eight different AT(N) biomarker profiles that can help to assign individuals into one of three categories 1) individual with normal AD biomarkers, 2) those in the AD continuum, and 3) those with normal amyloid but abnormal T or (N) (Jack et al., 2018). For those in the AD continuum he clinical progression of $\mathrm{AD}$ is understood to be on a continuum starting with preclinical $\mathrm{AD}$ and progressing to mild cognitive impairment (MCI) due to $\mathrm{AD}$, and finally dementia due to AD (Jack et al., 2018). During the preclinical phase of AD clinical symptoms such as memory loss are not apparent but early markers of AD are beginning to be present. Persons with $\mathrm{MCI}$ due to AD experience mild symptoms that do not interfere with everyday activities and typically demonstrate biomarker evidence of AD (Jack et al., 2018). The final phase on the AD continuum is dementia due to $\mathrm{AD}$ which can be further categorized into mild AD, moderate AD, and severe AD (Jack et al., 2018). The time patients spend in each phase varies and maybe affected by age, genetics, gender, as well as other factors ("2020 Alzheimer's disease facts and figures" 2020) . Further, 15-38\% of those with MCI may progress on to AD while others remain stable and few may revert back to normal cognition, the reasoning is not understood ("2020 Alzheimer's disease facts and figures" 2020. 


\section{Aims}

The overall aims of this program of research were to understand factors contributing to AD diagnosis variability in primary care practice settings and to find ways to improve early AD diagnosis by primary care providers. Four projects were undertaken to meet the aims and each resulted in a published manuscript.

The first project sought to describe the current evidence available to identify health care system factors that contribute to missed or delayed diagnosis of dementia by primary care providers through an integrative review of empirical studies identified through a systematic electronic search of the scientific literature databases (3. Romano R. R., Carter, Anderson, et al., 2020). The results were published in the Journal of the American Association of Nurse Practitioners. There was considerable variation in the diagnostic accuracy of dementia that could be attributed to barriers at the organizational, provider, and patient level.

The second project was published in the Journal of Alzheimer's Disease and compared the association between psychophysical responses to experimental thermal pain in males and females to determine if sex differences in pain exist across the cognitive spectrum (R. R. Romano et al., 2019). A secondary analysis of data from an age-and sex-matched between groups cross-sectional study suggested evidence that patterns of response to painful stimuli may differ between males and females with worsening cognition.

The third project was published in Biological Research for Nursing and was a narrative review of a systematic search of the scientific literature describing how sensory changes have been used as biomarker for AD (3. Romano R. R., Carter, and T. B. Monroe, 2020. The review found the most widely studied sensory system was olfaction and that changes in sense of smell, taste, vision, proprioception, and hearing are different between those with $\mathrm{AD}, \mathrm{MCI}$, or normal cognition.

The final project, which has been accepted for publication in Journal of Alzheimer's Disease, compared responses to painful thermal stimuli based on APOE $\epsilon 4$ allele status. As far as we know this is the first manuscript to make this comparison and we were able to demonstrate that those with an APOE $\epsilon 4$ allele were statistically more likely to experience a greater level unpleasantness of pain when compared to those without the epsilon 4 allele.

These four projects have provided a foundation for continuation in searching for ways in which research can assist primary care providers to improve their care to people living with $\mathrm{AD}$ and their families. The ultimate goal would be to identify those with AD or at least a high probability of developing $\mathrm{AD}$ well prior to the onset of symptoms.

The following four chapters provide the results of the projects that were undertaken for this dissertation. The chapters move from examining the practice characteristics that are related to primary care providers initiating screening, through the use of thermal 
responses across the cognitive spectrum, to understanding sensory changes to as potential biomarkers, and culminating with the differences in individuals with different APOE genes. 


\section{Chapter 2}

\section{Factors Influencing Dementia Detection in Primary Care Setting ${ }^{1}$}

\section{Background}

Alzheimer disease (AD) is the most common type of dementia and is estimated to affect over 5.7 million adults in the United States (Association, 2018). Alzheimer disease and other forms of dementia are often not detected until advanced stages of the disease because there is currently no definitive method for diagnosing AD except through direct observation of the brain after death (Association, 2018). Clinical symptoms of AD typically begin with subtle short-term memory problems or slight personality changes (Wilson et al., 2012), making early diagnosis difficult. Epidemiological studies suggest that at least half of the people living with AD have not yet received a diagnosis (Barker et al., 2002).

The prevalence of AD is expected to triple over the next 30 years (Hebert et al., 2013; Patterson, 2018). Estimated health care costs of AD in the United States were $\$ 277$ billion for 2018 and are estimated to surpass $\$ 1.1$ trillion by 2050 if there is no change in preventing or delaying AD (Association, 2018). Estimates suggest that slowing disease progression by at least 5 years is estimated to reduce the overall mortality rate, financial costs, and public burden related to $\mathrm{AD}$ by $39 \%$ (Zissimopoulos, Crimmins, and St Clair, 2014).

Less than half of persons with dementia and/or their caregivers report any history of clinical cognitive evaluation (Kotagal et al., 2015). One reason for low reports of receiving a cognitive evaluation is that the United States Preventive Services Task Force does not currently recommend screening for cognitive impairment in older adults, arguing that there is insufficient evidence to support the benefit of early detection particularly in light of ineffective treatment (Moyer and Force, 2014). However, the lack of effective treatment

\footnotetext{
${ }^{1}$ Reprinted from the final submission with permission. Romano R,R., 3rd, Carter M.A., Anderson A.R., et al.. An integrative review of system-level factors influencing dementia detection in primary care. J Am Assoc Nurse Pract. 2020;32.4, pp. 299-305. https://doi.org/10.1097/jxx.0000000000000230.
} 
may be changing because ongoing studies are testing novel drugs and non-drug therapeutic interventions that may help prevent the onset or delay the progression of the disease (Cummings et al., 2019) and current pharmacological and non-pharmacological interventions seem to be most beneficial during the earliest stages of AD (Robinson, Tang, and Taylor, 2015). Thus, early recognition of AD is critical for current and future treatment effectiveness.

There are other reasons that nurse practitioners (NPs) and other primary care providers may not screen for $\mathrm{AD}$. One reason contributing to limited screening for AD results from health care system factors minimizing the need for this service. For example, health care system models may constrain NP's practice behavior by failing to recognize how important early diagnosis is to individuals with AD and their families. Andersen's behavioral model of health service utilization suggests that environmental characteristics influence behavior (Andersen, 2008; Andersen, 2008). This model proposes the importance of understanding the relationships between environmental characteristics and screening behaviors by NPs caring for people with AD and their families. Future studies focused on improving screening behaviors must consider these environmental characteristics when designing, implementing, and evaluating the behavior-change intervention.

An older survey of 741 caregivers of people with AD found that $74 \%$ of families first approached their primary care provider with concerns about the memory of their loved one (Wilkinson et al., 2004). However, in the same study the authors found that in $70 \%$ of the cases, the diagnosis of $\mathrm{AD}$ was made by someone other than their primary care provider (Wilkinson et al., 2004). To our knowledge, this is the most recent study to address this phenomenon.

\section{Objective}

The overall goal of this literature review was to better understand why NPs may not routinely screen patients for AD. The specific objective was to identify factors in primary care practices reported in the literature that influence missed or delayed diagnosis of cognitive impairment. Understanding these factors will assist NPs in providing optimal care to patients with AD and their caregivers. Early detection of cognitive changes is necessary to trigger further evaluation leading to a formal diagnosis. This is not only critical to diagnosing dementia, but also for uncovering reversible causes of cognitive decline such as normal pressure hydrocephalus, thyroid disease, vitamin deficiency, or depression.

\section{Methods}

Studies were searched electronically through MEDLINE (OVID interface, 1948 onwards), the Cochrane Central Register of Controlled Trials (Wiley interface, current issue), CINAHL, 
and PsycINFO. The search strategy aimed to find peer-reviewed manuscripts that identified organizational, provider, or patient characteristics of the primary care setting that influenced screening in AD. A combination of three terms, "dementia" and "diagnosis" and "primary care," were used plus four purposefully selected search terms - "barrier," "attitude," "organizational factors," and "missed diagnosis"- were used in each electronic search. All potential English-language manuscripts were exported directly to EndNote.

Manuscripts were considered for inclusion if they met the following criteria: 1 . They were empirical studies; 2 . They identified factors for missed or delayed diagnosis; 3 . They included a comparison of diagnostic accuracy by primary care providers; or 4 . They identified organizational characteristics related to diagnosis of AD. Manuscripts were excluded for the following criteria: 1. They were not published in English; 2. They were reviews or commentaries; or 3. Full articles could not be obtained.

First, all duplicates were removed. Next, articles that were not topically related based on title and subsequent reading of the abstract were excluded. And third, articles that met the above exclusion criteria were removed (Figure 2.1).

A piloted data collection form was developed and revised to capture consistent information to address the research objective. The data collection form included information on: the estimated prevalence of dementia in the sample, the accuracy of provider diagnosis including cognitive impairment, dementia, Alzheimer's disease, vascular dementia, other diagnosis; the tools used by the provider to make an estimation of cognitive impairment; and the tool used to determine accuracy in diagnosis. Additionally, the form collected textual data on any factors identified that contributed to the diagnosis of AD or a related dementia. The overwhelming majority of the literature focused on "Alzheimer's Disease" or "dementia" and for the purposes of this paper we elected to use the term AD because it is the most common sub-type of dementia and there are specific diagnostic criteria for probable or possible AD.

Risk of bias in the remaining studies was assessed using design specific tools developed by the Risk Assessment Work Group of the National Heart, Lung, and Blood Institute (Study Quality Assessment Tools, 2020). The ratio was calculated for each study between point values (e.g., $\mathrm{No}=0$, Yes=1, Good=2, Fair=1, Poor=0) assigned for each item to total number of questions. Each author reviewed the scoring criteria and a final score consensus was reached by all authors. 

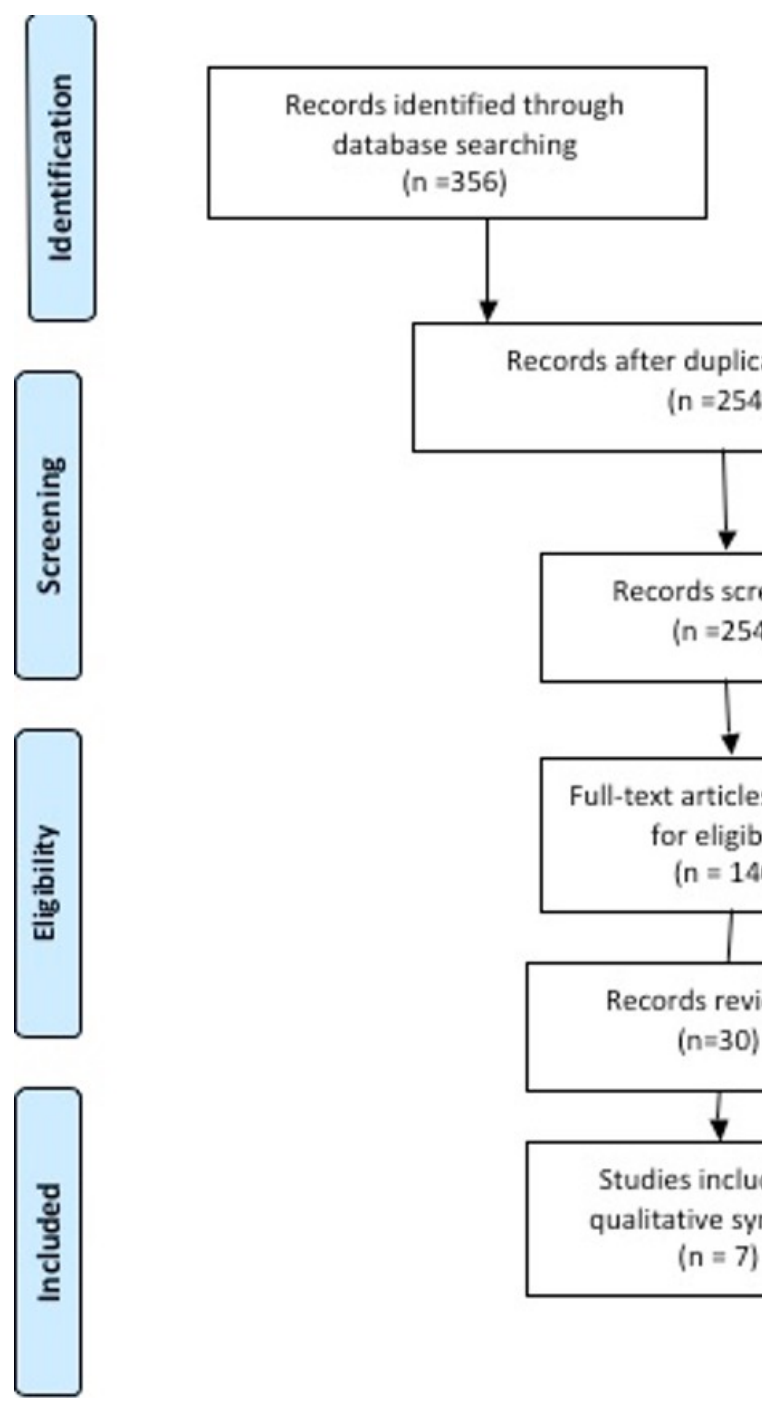
Additional records identified through other sources

$$
(n=8)
$$

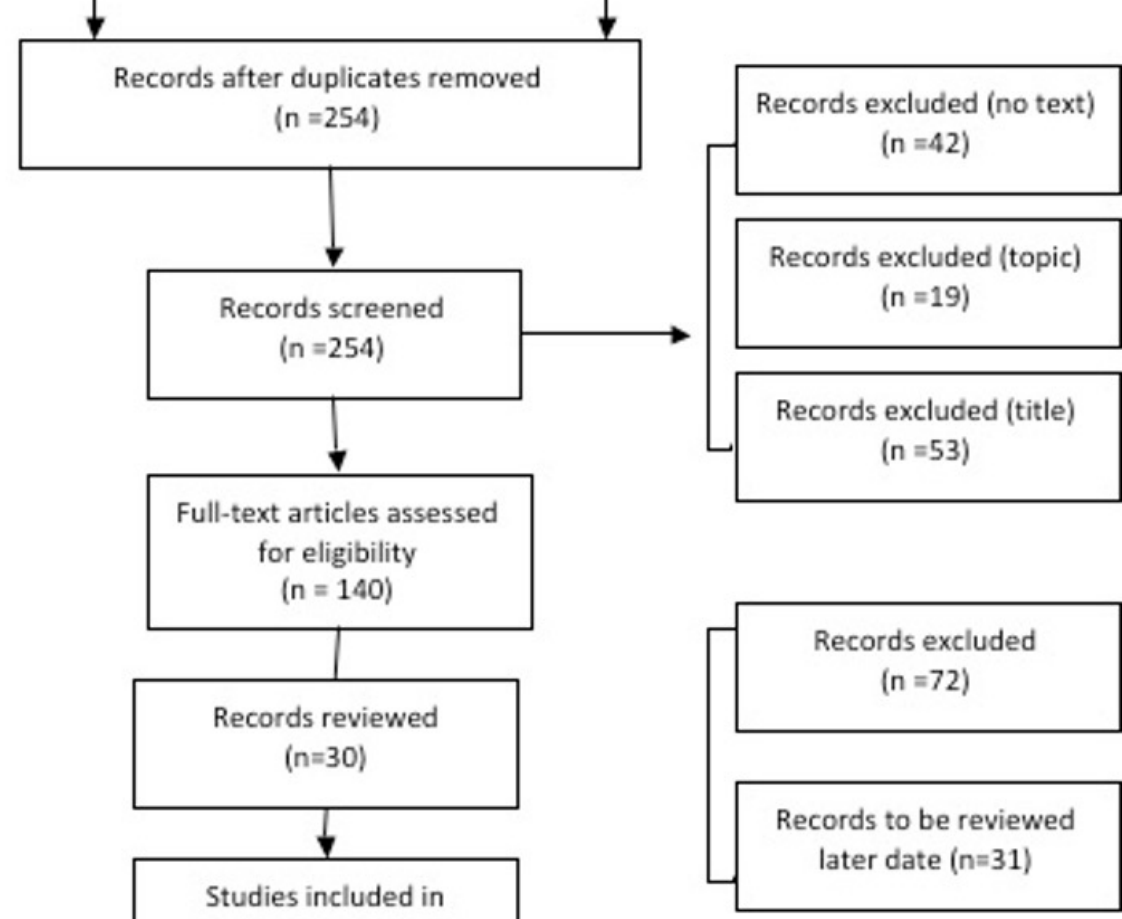

Figure 2.1: Consort prisma diagram. 


\section{Results}

The search yielded 140 empirical studies. Of these, 133 studies were excluded because they focused on the validation of specific screening tools, the detection of other illnesses, or dementia care outside primary care. This resulted in seven articles selected to be included in the literature review: two were interventional studies, two were case-comparison studies, and three were cross-sectional studies (Table 2.1). None of these studies examined NP practices specifically and it is likely that few, if any, NPs were included in the sample. A comparison of study characteristics is provided in Table 2.1. Most studies were rated as high quality, with the weakest study receiving a calculated score of 0.52 (range 0-2) using the bias risk tool described above (Table 2.2. The major threat to internal validity was selection bias across all studies as most studies relied on non-random methods to select participants. A description of internal threats to validity are presented in Table 2.2.

The studies estimating diagnostic accuracy after intervention are summarized in Table 2.3. Two studies compared the provider's clinical judgment against a neuropsychological test battery, while one study compared clinical diagnosis to autopsy results. Prevalence estimated by clinicians ranged from $6-51 \%$ and the accuracy of diagnosis was between $19-81 \%$. Identifying factors contributing to the detection of dementia are summarized in Table 2.4.

The most widely cited organizational barrier was lack of services, while the most widely cited provider barrier was lack of the provider's ability to accurately make the diagnosis. A frequently noted patient barrier to accurate diagnosis of dementia was the occurrence of confounding comorbidities or frailty. 
Table 2.1: A descriptive comparison of studies.

\begin{tabular}{lllcll}
\hline Author & Country & Population & N & Study Type & Theme \\
\hline Venezian (2016) & Italy & Provider & 131 & Cross-sectional & Knowledge of AD \\
Magin (2016) & Australia & Patients & 489 & Cross-sectional & Attitudes towards AD \\
Edwards (2015) & England & Providers & 28 & Intervention & Improve patient- \\
& & Care Staff & 62 & & centered outcomes \\
Tierny (2014) & Canada & $\begin{array}{l}\text { Providers } \\
\text { Patients }\end{array}$ & 13 & Case comparison & Accuracy of diagnosis \\
Fortinsky (2010) & USA & Providers & 422 & Cross-sectional & Geographic variation \\
Boustani (2005) & USA & Patients & 3340 & Quality & Implementing screening \\
& & & & improvement & protocol \\
Mok (2004) & USA & Patients & 463 & Case comparison & Accuracy of diagnosis \\
\hline
\end{tabular}

Table 2.2: Descriptive comparison of studies quality.

\begin{tabular}{|c|c|c|}
\hline Author (Year) & $\begin{array}{l}\text { Quality } \\
\text { Score }\end{array}$ & Threats to Internal Validity \\
\hline \multicolumn{3}{|l|}{ Cross-Sectional } \\
\hline Veneziani (2016) & 0.76 & $\begin{array}{l}\text { History threat (other surveys), selection bias (younger females), } \\
\text { social desirability }\end{array}$ \\
\hline Magin (2016) & 0.79 & Selection bias (non-random sample, selected by receptionist) \\
\hline Fortinsky (2010) & 0.83 & Possible selection bias (39\% response rate) \\
\hline \multicolumn{3}{|l|}{ Case-comparison } \\
\hline Tierney (2014) & 0.80 & Selection bias (non-random sample) \\
\hline Mok (2004) & 0.91 & $\begin{array}{l}\text { Selection bias (non-random selection of participants), } \\
\text { maturation bias (better able to access autopsy) }\end{array}$ \\
\hline \multicolumn{3}{|l|}{ Interventional } \\
\hline Edwards (2015) & 0.75 & Potential testing bias (pre-test cues), Potential maturation bias \\
\hline Boustani (2005) & 0.52 & $\begin{array}{l}\text { Instrumentation bias (false negatives not accessed, no clinical data support), } \\
\text { selection bias (large number did not participate) }\end{array}$ \\
\hline
\end{tabular}

* Ratio of the total score determined using NIH study quality assessment tools and the number of items assessed. Author consensus reaching in support of scoring.

Table 2.3: Descriptive comparison of accuracy of dementia diagnosis.

\begin{tabular}{lllcc}
\hline Study & Provider Method & Referenced Method & Prevalence & Accuracy \\
\hline Tierney (2014) & Clinical judgment & Neuropsychological test battery & $30 \%$ & $60.6 \%$ \\
Boustani (2005) & Clinical judgment & 6-item screener, CSI-D & $6 \%$ & $18.5 \%$ \\
& & $\begin{array}{l}\text { CERAD battery, GDS } \\
\text { semi-structured interview }\end{array}$ & $51 \%$ & $81.2 \%$ \\
\hline
\end{tabular}


Table 2.4: Factors identified contributing to detection and care of dementia.

\begin{tabular}{|c|c|c|c|c|c|c|c|}
\hline Factors & $\begin{array}{c}\text { Veneziani } \\
\text { (2016) }\end{array}$ & $\begin{array}{c}\text { Magin } \\
(2016)\end{array}$ & $\begin{array}{c}\text { Edwards } \\
\text { (2015) }\end{array}$ & $\begin{array}{l}\text { Tierney } \\
\text { (2014) }\end{array}$ & $\begin{array}{c}\text { Fortinsky } \\
\text { (2010) }\end{array}$ & $\begin{array}{c}\text { Boustani } \\
\text { (2005) }\end{array}$ & $\begin{array}{l}\text { Mok } \\
(2004\}\end{array}$ \\
\hline \multicolumn{8}{|l|}{ Organizational } \\
\hline Location & & & & & $x$ & & \\
\hline Time & & & & & $x$ & & \\
\hline Lack of services & $\mathrm{x}$ & & $\mathrm{x}$ & & & & \\
\hline Lack of specialists & $\mathrm{x}$ & & & & & & \\
\hline Lack of support staff & & & & & $\mathrm{X}$ & & \\
\hline Lack of reimbursements & & & & & $x$ & & \\
\hline Patient load & & & & $x$ & $\mathrm{X}$ & & \\
\hline \multicolumn{8}{|l|}{ Provider } \\
\hline Gender & $\mathrm{x}$ & & & & & & \\
\hline Education & $\mathrm{x}$ & & $\mathrm{x}$ & & $x$ & & $x$ \\
\hline Attitudes & & & $\mathrm{x}$ & & & & \\
\hline Testing process & $\mathrm{x}$ & & & & & $\mathrm{X}$ & \\
\hline Communication & & & $\mathrm{X}$ & & & & \\
\hline \multicolumn{8}{|l|}{ Patient } \\
\hline Age & & $\mathrm{x}$ & & & $x$ & & \\
\hline Gender & & & & & $\mathrm{X}$ & & \\
\hline Severity/Risk/Comorbidities & & $\mathrm{x}$ & $\mathrm{x}$ & $x$ & & $x$ & \\
\hline Awareness & & $\mathrm{x}$ & & $x$ & & & \\
\hline Attitudes & & $\mathrm{x}$ & & & & & \\
\hline
\end{tabular}




\section{Discussion}

This review of research literature suggests that the clinical presentation of dementia is heterogenous and results in variation in diagnostic accuracy. Results indicate that primary care providers have varying ability to accurately diagnose AD irrespective of education and training (Association, 2018; Mok et al., 2004; Tierney et al., 2014). The study by Mok et al., 2004 suggested that diagnostic accuracy was $84 \%$ when confirmed via autopsy among a group of community based primary care providers. Similarly, Tierney et al., 2014 reported the specificity of clinical judgment to detect $\mathrm{AD}$ was $86 \%$ in primary care providers. Tierney et al., 2014 concluded that many provider and patient characteristics influenced the likelihood of detecting dementia, and that providers seeing a greater number of cognitively impaired individuals demonstrated better clinical judgment. The authors further noted that overall complexity of dual comorbidities heavily influenced diagnostic accuracy (Tierney et al., 2014). The ability to accurately discern cognitive impairment was not associated with routinely conducting cognitive assessments on individuals with positive family history or frequently asking about cognitive function on exam (Tierney et al., 2014).

Similarities in the results from Mok et al., 2004 and Tierney et al., 2014 suggest that organizational need, such as higher patient burden, may be a greater factor influencing accuracy in diagnosis and may be an influencing factor on practice behavior. No intervariable correlations were presented by Tierney et al., 2014 but it may be possible that NPs will increase their screening for $\mathrm{AD}$ as the number of patients with dementia in the practice increases.

Provider educational needs have been identified as a major factor at both the provider and patient level to diagnosis of AD (R. Edwards, Voss, and Iliffe, 2015; Fortinsky et al., 2010H; Martin, Fleming, et al., 2015; Veneziani et al., 2016). Provider attitude towards dementia care and treatment are also major factors influencing the accurate detection and diagnosis of dementia (Table 2.4). An educational intervention developed for primary care providers showed an improvement on pre-test versus post-test measures on both knowledge and attitudes, suggesting that knowledge is necessary to improve attitudes ( $\mathrm{R}$. Edwards, Voss, and Iliffe, 2015).

Although improvements were seen on overall attitude scores, providers continued to associate lack of diagnosis with lack community support and services (R. Edwards, Voss, and Iliffe, 2015). In a different study, the provider practice of referring patients with cognitive impairment to community support services varied by geographic location, further suggesting that organizational factors contribute to the detection and care of dementia (Fortinsky et al., 2010H).

Magin et al., 2016 reported that the majority of patients would like to be tested for dementia. However, lack of comfort communicating with patients about dementia and disclosing diagnosis was found by Veneziani et al., 2016 to be a contributing barrier 
to diagnosis. Interestingly, Veneziani and colleagues found that the routine practice of administering cognitive screening tests did contribute to overall knowledge and attitude about dementia (Veneziani et al., 2016). Additionally, results found by Tierney et al., 2014, found that routine cognitive screening does not contribute to the ability of providers to accurately discern pathological cognitive impairment.

Findings by Boustani et al., 2005 support that a screening protocol alone is not sufficient to improve identifying patients with $\mathrm{AD}$. In a practice with a prevalence of $6 \%$ dementia, less than half ( $47 \%)$ of the patients with dementia were identified by a screening protocol. The researchers concluded that a more complex intervention may be warranted. This intervention should consider all levels of the health care system (Boustani et al., 2005).

\section{Gaps in Literature}

We found no study that explicitly measured data related to organizational characteristics such as staffing levels. Only one study explored self-reported organizational level factors including reimbursement, time constraint, and community resources and did not conduct an objective, independent assessment of these factors (Fortinsky et al., 2010H). Review findings suggest that organizational and environmental characteristics contribute to detection and care of dementia, yet no study directly used organizational structures as the main predictive variable.

A larger gap exists in the evidence to understand the reasons behind discordant diagnosis, although education is constantly cited as the reason. Educational needs are an obvious barrier, although Veneziani et al., 2016, did not find an association between year of graduation of the provider and attitude or knowledge. However, other studies have demonstrated this relationship (Boise et al., 1999; Boise, 2006; Bradford et al., 2009). The lack of association in the Veneziani et al., 2016, study may be the result of selection bias and lack of generalizability as the sample was drawn from a research network (Veneziani et al., 2016). In addition, only three studies identified in this literature search explored how electronic health record decision support systems impacted dementia care and diagnostic accuracy (Chou et al., 2012; Eichler et al., 2014; Grypma, 2007).

\section{Limitations}

The future goal of this work is to inform research about how AD screening can be implemented and evaluated by primary care NPs. This review has three main limitations. The first limitation is that one researcher conducted the literature search, review of articles, and extraction of data. To minimize this limitation, the reviewer adhered to a coding sheet and revisited articles after any edits were made to the coding sheet. Also, the authors used a published quality scale and reached consensus in the evaluation of each study, helping to reduce bias in the results. 
The second limitation is the heterogeneity of diagnosis and prevalence across the articles. We did not attempt to control for this heterogeneity, therefore, interpretation of diagnostic accuracy may be skewed. Not surprisingly, the majority of articles focus on $\mathrm{AD}$ or "dementia" with little attention given to the diagnosing specific sub-types of dementia. However, this limitation is overcome by the overwhelming majority of dementias presenting with some form of pathologic cognitive impairment, which is a key component to accurate diagnosis. The third limitation is that none of the articles reported whether or not NPs were included in the sample or how NP practices might be different from other providers. Multiple studies have reported that NPs and physicians have similar practice outcomes, but this may not hold for screening for AD (Stanik-Hutt et al., 2013).

\section{Conclusions}

Primary care providers, and especially NPs, are the first point of contact for patients and families with concerns about memory loss. At the same time, patients presenting to health care settings with memory complaints tend to not perform well on cognitive measures (Kumar, Singh, and Ekavali, 2015). As the prevalence of AD is expected to increase and new, effective treatments are developed, there is a need to develop primary care services that detect AD earlier (Hebert et al., 2013). Dementia in general is under diagnosed, and major initiatives in the United States are aimed at improving diagnosis. For example, the National Institute on Aging in the National Institutes of Health has very useful materials on assessing cognitive impairment (Association, 2018), and a required part of Medicare's Annual Wellness Visit includes an assessment of cognition. The results of this review, however, suggest that solely implementing a screening protocol is not enough to improve identification of AD.

NPs are poised to be the frontline leaders in screening for pathologic cognitive impairment as well as previously mentioned known reversible causes of cognitive impairment (e.g., thyroid hormone alterations, normal pressure hydrocephalus, vitamin deficiency, severe depression, and adverse effects from some medications). Better knowledge by providers of reversible causes of cognitive decline could help address patient and provider level discomfort in diagnosing AD or a related dementia only addresses one component of the issue. Importantly, findings from the current review indicate that additional interventions that improve support systems, increase services for individuals with AD and their families, and clarifying testing procedures may be a more critical step in achieving the goal of earlier diagnosis. Finally, evidence presented in this review suggests that organizational and environmental factors exist that influence provider practice, and thus screening and diagnosing of dementia in primary care and these factors need to be explored further. 


\section{Chapter 3}

\section{Cognitive Function with Perceptions of Pain: Sex Differences in Seniors ${ }^{1}$}

\section{Introduction}

Nearly 50 million adults worldwide over the age of 65 years will develop dementia (Hebert et al., 2013; Patterson, 2018), and amongst those, an estimated 50-93\% will experience chronic pain (Abdulla et al., 2013; Corbett et al., 2014; Stubbs et al., 2016; Kooten et al., 2015). Dementia is a known risk factor for the under-treatment of pain (T. B. Monroe, Carter, et al., 2013; T. B. Monroe, Gore, Chen, et al., 2012; C. M. Paulson, T. Monroe, and Mion, 2014; Stubbs et al., 2016). People with Alzheimer's disease (AD) receive less pain medication for similarly painful conditions when compared with cognitively intact older adults (Haasum, Fastbom, Fratiglioni, Kareholt, et al., 2011; Haasum, Fastbom, Fratiglioni, and Johnell, 2012). Pain is a multidimensional experience arising from cognitive, sensory and affective central nervous system processes involving multiple neuronal circuits and brain regions.

Furthermore, AD impacts brain structure, such as the hypothalamus and the prefrontal cortex which is responsible for affective pain (Cole et al., 2006; A. K. Jones, Kulkarni, and Derbyshire, 2003; Scherder, Sergeant, and Swaab, 2003; Stubbs et al., 2016). A potential cause of the under-treatment of pain in dementia patients is the lack of understanding about how dementia influences the psychophysiological factors of the pain experience (Stubbs et al., 2016) and how these influences differ by sex in communicative people across the cognitive spectrum. Pain is the primary reason people seek medical attention, and more evidence is needed to understand the altered pain experience in AD patients as the prevalence of Alzheimer's disease and related dementias is expected to triple over the next 30 years (Hebert et al., 2013; Johannes et al., 2010; Patterson, 2018).

\footnotetext{
${ }^{1}$ Reprinted from final submission. Romano, R.R., Anderson, A.R., Failla, M.D., Dietrich, M.S., Atalla, S., Carter, M.A., Monroe, T.B., Sex Differences in Associations of Cognitive Function with Perceptions of Pain in Older Adults, 715-722., Copyright (2019), with permission from IOS Press. The publication is available at IOS Press through http://dx.doi.org/10.3233/JAD-190142
} 
Psychophysical studies of pain frequently report sex-related differences in the pain experience in cognitively healthy adults (Fillingim et al., 2009; P. E. Paulson et al., 1998; Straube et al., 2009). These sex differences may change with age since the prevalence of chronic pain increases with age as the function of endogenous pain inhibitory systems declines (R. R. Edwards, Fillingim, and Ness, 2003; Naugle et al., 2013; Riley et al., 2010). In cognitively healthy older adults, women tend to experience pain at a higher intensity and report pain more frequently than men (Johannes et al., 2010; Leveille et al., 2005). However, this relationship has not been demonstrated to exist across the cognitive spectrum.

Understanding patterns of pain responsivity across age, sex, and cognitive ability are essential given evidence of sex-associated differences in the psychophysical and neuropsychological processing of pain, the increased incidence of chronic pain in older adults, and the potential for undertreatment of pain in dementia. We have published several papers from the parent data set showing sex differences in cognitively healthy older adults (T. B. Monroe, Fillingim, et al., 2018). We have also shown sex differences in a pilot sample of people with dementia only (Cowan et al., 2017). To extend upon this work, we now examine sex differences in pain across the cognitive spectrum. While several between groups psychophysical studies on sex-differences in pain appear in literature, there is a paucity of experimental pain research using a continuous measure of cognitive ability to predict pain outcomes across the cognitive spectrum. Understanding patterns of experimental pain responsivity across age, sex, and cognitive ability are essential given evidence of sex-associated differences in the psychophysical and neuropsychological processing of pain. This understanding may help to develop more effective treatment plans for the growing number of older adults experiencing chronic pain with comorbid dementia. Thus, we tested the hypothesis that sex-differences in psychophysical processing of pain are significantly associated with increasing or decreasing levels of global cognition.

\section{Methods}

\section{Study Design}

This study is a secondary analysis of a cross-sectional parent study designed to compare the psychological response to thermal pain in older adults with and without dementia. Thus, the current methods have been previously published (Cowan et al., 2017; T. B. Monroe, Beach, et al., 2017). The purpose of the current study was to examine sex differences in the association of cognitive impairment and thermal pain perception in verbally communicative older adults. Cognitive impairment, as assessed by the MMSE (range 0-30), was used as the primary co-variate in our outcome analysis and included scores across the cognitive spectrum (no cognitive impairment to severe cognitive impairment).

The Vanderbilt University Institutional Review Board approved the parent study, 
and each participant, or legal surrogate when appropriate, provided written informed consent at the time of enrollment. Capacity to consent was assessed using the University of California San Diego Brief Assessment of Capacity to Consent (UBACC), that takes less than 5 minutes to complete. A UBACC score higher than 14.5 was required for informed consent, as this cut-off is $89 \%$ sensitive and $100 \%$ specific for determining capacity to consent for research (Jeste et al., 2007). Individuals with UBACC scores less than or equal to 14.5 (i.e., those lacking decisional capacity) signed an assent document and a legal surrogate provided consent. All participants and legal surrogates who completed the study were compensated US $\$ 100$ for their time.

\section{Participants}

The recruitment and enrollment protocol of the parent study are previously published (Cowan et al., 2017; T. B. Monroe, Beach, et al., 2017). Participants 65 years of age or older were recruited from the greater Nashville, Tennessee metropolitan area and enrolled over three years from 2012 to 2015. A clinical diagnosis of AD was verified based on documentation in the medical record of 1) neuropsychiatric evaluation, diagnostic MRI or PET, lack of other potential causes of memory loss; and 2) results of the evaluation with the MMSE, Montreal Cognitive Assessment or the Functional Assessment Staging Scale. Cognitively healthy participants were recruited using a mass email, flyers, and recruitment presentations in healthcare facilities and local events.

Participants were excluded for the following reasons: the presence of a chronic pain diagnosis, daily use of opioid or non-opioid pain medication, upper extremity peripheral neuropathy, any current cancer diagnosis or treatment, previous stroke with residual deficit, Raynaud's Disease, unstable cardiac conditions, insulin-dependent diabetes, or current diagnosis of major depression.

Because the parent study included neuroimaging, additional exclusion criteria included claustrophobia, the presence of a pacemaker, ventricular shunt, or any implanted metal object not confirmed as 3 Tesla MRI compatible, multiple metal implants in the same extremity, or presence of movement disorders (e.g., Parkinson's disease, Restless Leg Syndrome). Participants were required to be verbally communicative and able to provide a verbal pain rating.

\section{Screening and Enrollment}

A two-part screening process included an initial telephone screening, followed by a onehour visit to confirm eligibility either at the participant's place of residence or at the Vanderbilt University Medical Center. Before any data collection occurred participants and the legal surrogate (when necessary) were allowed to experience the thermal pain stimulus and complete two practice psychophysics trials. Practice trials ensured that participants 
understood the directions and could respond appropriately. Evidence of an appropriate response included the ability to follow directions and vocalize pain.

\section{Measures}

A trained research assistant administered all study measures orally to participants to decrease subject and caregiver burden and to minimize missing data. Demographic measures included a detailed list of all medications and the Hollingshead Four-Factor Index of Socioeconomic Status (SES) (Hollingshead, 1975). The Brief Pain Inventory Short Form (BPI-SF) was used to collect current and average daily pain (Cleeland and Ryan, 1994). Depression and anxiety screens included the Geriatric Depression Scale-Short Form (GDS15) (Kurlowicz, 1999) and the state and trait forms of the Spielberger State-Trait Anxiety Inventory (STAI) (Spielberger, 1971), respectively.

\section{Thermal Stimulation Protocol}

Trained research assistants administered the psychophysical thermal stimulation assessment over approximately 30 minutes following a modified protocol of the experimental mechanical pressure pain protocol by Cole et al., 2006. Two aspects of pain were assessed using the Medoc Pathway Pain and Sensory Evaluation System: the intensity, how strong the pain feels; and the unpleasantness, how unpleasant or disturbing the pain feels. A 30 x $30 \mathrm{~mm}$ Medoc thermode was attached to the thenar eminence of the right hand. The thermode was programmed to deliver a baseline temperature of $30^{\circ} \mathrm{C}$ with increasing heat at a rate of $1^{\circ} \mathrm{C} / \mathrm{s}\left(\max\right.$ temperature $\left.=48^{\circ} \mathrm{C}\right)$.

Participants were instructed to signal to stop the increasing heat when the participant felt 'warmth,' 'mild pain,' or 'moderate pain. The temperatures at which each participant stopped the increasing heat at each percept were documented. Following the approach we have previously published (Cowan et al., 2017; T. B. Monroe, Beach, et al., 2017), pain intensity was evaluated using a 0-20 sensory numeric rating scale (Figure 3.1) with anchors "warmth=0," "mild pain=5," and "moderate pain=11." Unpleasantness was evaluated using a 0-20 unpleasantness scale with anchors " $0=$ neutral," " $5=$ slightly unpleasant," "8=unpleasant," "11=very unpleasant," "16=intolerable," and "20=extremely distressing." Participants were acclimated to the scales and then completed three trials at each temperature condition, and the average self-reported pain intensity and unpleasantness ratings across the trials used in our analyses.

\section{Statistical Analysis}

Participant characteristics and study data collected on a continuous scale were summarized using median and inter-quartile range (IQR) due to lack of normality (Fischer test $> \pm 2.58$ ). Frequency distributions summarized nominal and ordinal data. Comparisons between males and females were conducted using Mann-Whitney $U$ tests (continuous) 


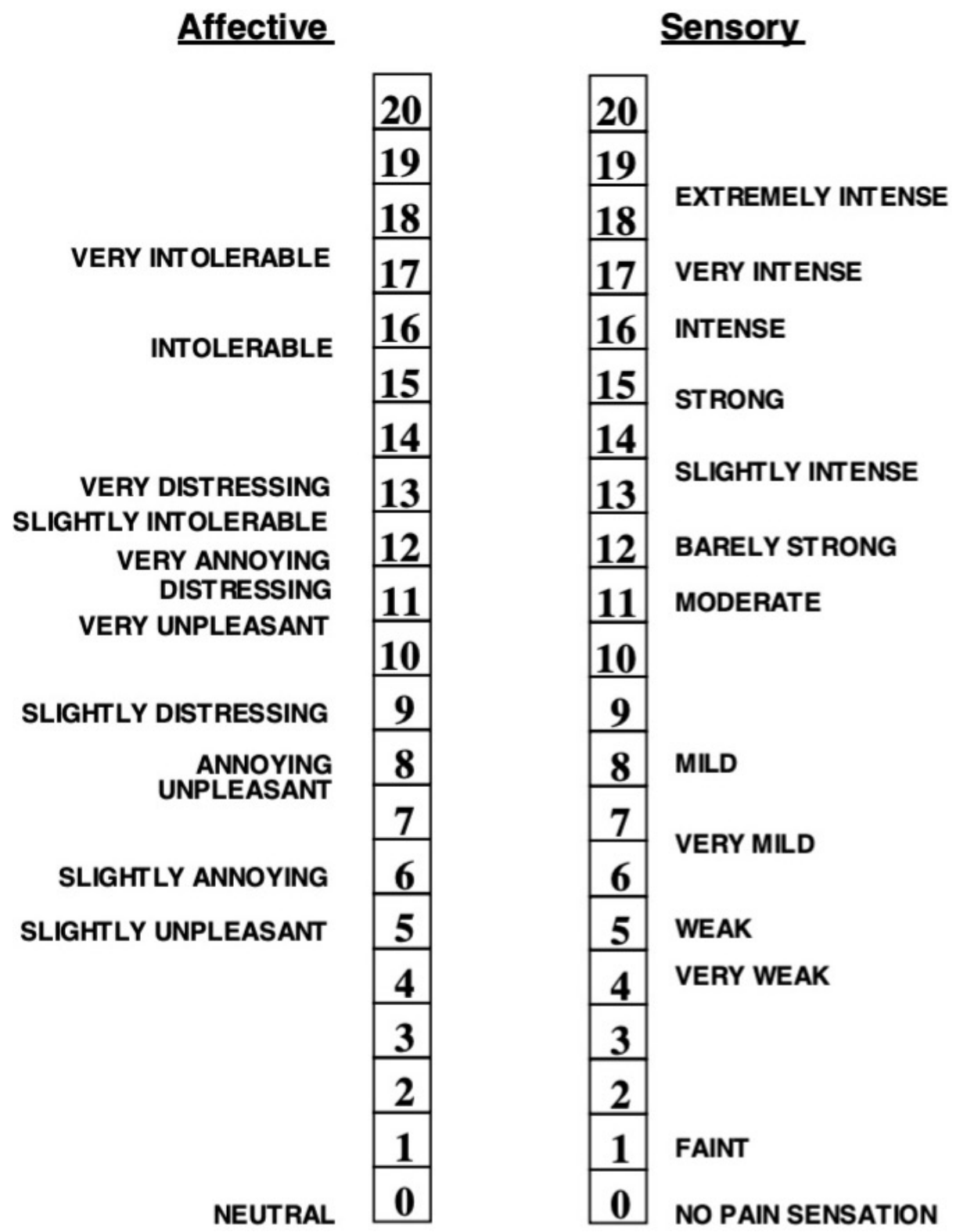

Figure 3.1: Numerical descriptor scale used to measure affective unpleasantness and sensory intensity. Reprinted from Eur J Pain, 9, Petzke F, Harris RE, Williams DA, Clauw DJ, Gracely RH, Differences in unpleasantness induced by experimental pressure pain between patients with fibromyalgia and healthy controls. 325-335. Copyright (2005), with permission from John Wiley and Sons. 
and Chi-Square Tests of Independence (nominal, ordinal). Cohen's d effect size indices were generated to summarize the magnitude of the gender effects. Associations of global cognition (MMSE scores) with the psychophysical reports (temperatures and ratings of unpleasantness) for males and females were generated using Pearson correlations. Differences between males and females in the strength and/or direction of those associations were tested using linear regressions. Each of the gender and MMSE distributions were mean centered and multiplied to create an interaction term that was entered into each regression, along with gender and MMSE as main effects, to test for differences between males and females in the association of MMSE with the respective psychophysical report used in the regression.

Before conducting these correlations and regressions, the skewed psychophysical distributions were transformed to normal. Such that at the warmth threshold, both temperature and unpleasantness values were rank transformed and at the mild and moderate pain thresholds, unpleasantness values were square-root transformed. For all analyses, a maximum alpha of $0.05(\mathrm{p}<0.05)$ was used for determining statistical significance.

\section{Results}

\section{Demographics}

A total of 80 participants were in the parent study. For this analysis, three participants were excluded due to an MMSE score of less than 10 and one subject was excluded due to missing an MMSE score. The final sample consisted of males $(\mathrm{N}=38)$ and females $(\mathrm{N}=38)$ with a median age of 73 (IQR: 68-80). MMSE scores (Table 3.1) ranged from 11 to 30 (IQR: 20-30). Of the sample $84 \%$ were white. On the day of the study, very few participants reported any pain (median both average and now $=0$ ) and fewer than $10 \%$ reported pain levels of greater than 2 ( 7 of $76,9 \%)$. There were no statistically significant differences between males and females on any of the participant characteristics investigated including most importantly the distribution of MMSE scores (Table 3.2).

\section{Psychophysical}

Summaries of the psychophysical self-report data for males and females are in Table 3.3. Females reported mild and moderate pain at statistically significantly lower temperature values than did males $(\mathrm{p}<.05$, Cohen's $d=0.51-0.54)$. Males, on the other hand, rated the experience of moderate pain statistically significantly more unpleasant than did the females ( $p=.016$, Cohen's $d=0.57)$. 
Table 3.1: Distribution of MMSE by gender groups.

\begin{tabular}{lcc}
\hline MMSE$^{\mathbf{1}}$ & $\begin{array}{c}\text { Female } \\
(\mathbf{n = 3 8 )}\end{array}$ & $\begin{array}{c}\text { Male } \\
(\mathbf{n}=\mathbf{3 8})\end{array}$ \\
\hline Cognitively Intact (29-30) & 14 & 17 \\
$\mathrm{MCI}^{2}(25-28)$ & 8 & 6 \\
Mild (20-24) & 9 & 5 \\
Moderate (15-19) & 1 & 6 \\
Moderately Severe (11-15) & 6 & 4 \\
\hline${ }^{1}$ MMSE-Folstein Mini Mental State Examina- \\
tion (range=0-30; 0=completely cognitively \\
impaired 30=completely cognitively intact). \\
2 MCI- mild cognitive impairment.
\end{tabular}

\section{Associations of MMSE with Psychophysical Reports}

Associations of the participant MMSE scores with their psychophysical reports (temperature and unpleasantness) are summarized for the entire sample and by sex in Table 3.4. A statistically significant inverse association of MMSE with temperature reports at the warmth threshold was observed for both males and females $(r=-0.33$ and -0.41 respectively, $\mathrm{p}<.05)$ with no statistically significant difference between them $(\mathrm{p}=.767)$. Thus, regardless of sex, temperatures at which a warmth threshold was reported increased as MMSE scores decreased. Minimal associations were detected between MMSE scores and temperature reports at either of the other two thresholds for both sex groups $(r=-0.13$ to $-0.05, \mathrm{p}>.50$, (Table 3.4).

There was a statistically significant difference between males and females in the direction of the association of MMSE scores with unpleasantness ratings at the moderate pain threshold $(\mathrm{p}=.033)$. In females, the direction of the association was that ratings of unpleasantness increased as MMSE scores increased $(r=0.30, p=.072)$. To the contrary, in males the direction of the association was reversed; ratings of unpleasantness at that threshold increased as MMSE scores decreased $(\mathrm{r}=-0.20, \mathrm{p}=.221)$. Thus, while neither of the specific correlations within each sex group was statistically significant, the magnitude of the difference between them in terms of the direction (positive or inverse) was statistically significant (interaction term, $\mathrm{p}=.033$ ). None of the other associations between MMSE scores and unpleasantness reports at either of the other two thresholds (warmth or mild pain) for both sex groups were statistically significant $(r=-0.19$ to $+0.07, p>.20)$ nor were any of the tests of difference between those correlations (Table 3.4). 
Table 3.2: Demographic and clinical characteristics of the participants.

\begin{tabular}{|c|c|c|c|c|}
\hline Characteristic & $\begin{array}{c}\text { Total } \\
(\mathrm{n}=76)\end{array}$ & $\begin{array}{c}\text { Female } \\
(n=38)\end{array}$ & $\begin{array}{c}\text { Male } \\
(n=38)\end{array}$ & P-value \\
\hline & & N (\%) & & \\
\hline \multicolumn{5}{|l|}{ Race } \\
\hline White & $64(84)$ & $31(82)$ & $33(878)$ & .529 \\
\hline \multicolumn{5}{|c|}{ Median [IQR] } \\
\hline Age & $73[68,80]$ & $73[68,80]$ & $73[67,81]$ & .731 \\
\hline $\mathrm{MMSE}^{1}$ & $28[20,30]$ & $27[21,30]$ & $28[18,30]$ & .887 \\
\hline BPI-average ${ }^{2}$ & $0[0,2]$ & $0[0,3]$ & $0[0,2]$ & .298 \\
\hline BPI Now ${ }^{2}$ & $0[0,0]$ & $0[0,0]$ & $0[0,0]$ & .893 \\
\hline $\mathrm{GDS}^{3}$ & $1[0,4]$ & $2[0,4]$ & $1[0,3]$ & .929 \\
\hline STAI State ${ }^{4}$ & $48[44,50]$ & $50[45,53]$ & $47[45,50]$ & .105 \\
\hline STAI Trait ${ }^{4}$ & $47[44,50]$ & $47[43,50]$ & $47[44,49]$ & .877 \\
\hline $\mathrm{SES}^{5}$ & $54[41,61]$ & $53[38,58]$ & $55[45,62]$ & .324 \\
\hline
\end{tabular}

${ }^{1}$ MMSE-Folstein Mini Mental State Examination (range $=0$ 30; $0=$ completely cognitively impaired $30=$ completely cognitively intact)

2 BPI-SF-Brief Pain Inventory Short Form (range=0-10; $0=$ no pain, $10=$ most pain)

${ }^{3}$ GDS-SF-Geriatric Depression Scale Short Form (range; $0=$ no indication of depression, 15=high possibility of depression); Female: $n=37$, Male: $n=37$

4 STAI-Spielberger State or Trait Anxiety Inventory (range; $20=$ indicate increased anxiety, $80=$ indicates least amount of anxiety); Female: $n=34$, Male: $n=32$

${ }^{5}$ Hollingshead Four Factor Measure of Socio-Economic Status (range=8-66; 8=lowest SES, 66=highest SES) Female: $\mathrm{n}=38$, Male: $\mathrm{n}=36$ 
Table 3.3: Psychophysics of temperature thresholds necessary to produce warmth, mild pain, or moderate pain and unpleasantness ratings at each condition.

\begin{tabular}{lcccc}
\hline Psychophysics & $\begin{array}{c}\text { Female } \\
(\mathbf{n}=\mathbf{3 8})\end{array}$ & $\begin{array}{c}\text { Male } \\
\mathbf{( n = 3 8 )}\end{array}$ & P-value & Cohen's d \\
\hline \multicolumn{5}{c}{ Median[IQR] } \\
Temperature ${ }^{1}$ & & & \\
Warmth & $33[32,35]$ & $33[32,36]$ & .139 & 0.34 \\
Mild Pain & $35[35,40]$ & $39[35,44]$ & .022 & 0.54 \\
Moderate Pain & $39[38,43]$ & $44[37,46]$ & .029 & 0.51 \\
Unpleasantness ${ }^{2}$ & & & \\
Warmth & $0[0,1]$ & $0[0,2]$ & .115 & 0.30 \\
Mild Pain & $3[0,5]$ & $4[1,6]$ & .140 & 0.33 \\
Moderate pain & $5[2,8]$ & $7[5,11]$ & .016 & 0.57 \\
\hline${ }^{1}$ Temperature was measured in degrees Celsius (range $=30-55$ \\
${ }^{\circ}$ C). \\
${ }^{2}$ Unpleasantness was measured by numerical descriptor scale $(0$ \\
$=$ neutral, 20 = extremely unpleasant).
\end{tabular}




\section{Discussion}

We found in both males and females that those with a lower global cognition rating required a higher temperature to elicit the perception of pain and males tended to report greater unpleasantness. This result parallels our previously reported pilot study (Cowan et al., 2017) from this sample in which males with AD only, reported pain as more unpleasant. This study extends these findings in individuals across the cognitive spectrum. Irrespective of cognitive status in the parent study, males reported pain as more unpleasant. These results are important because they demonstrate that sex differences in the perception of pain might be present across the cognitive spectrum.

Our finding that a greater pain stimulus is required to elicit the perception of pain with worsening cognition agrees with several previous studies from our laboratory and others (Cole et al., 2006; Kunz, Scharmann, et al., 2007; T. B. Monroe, Carter, et al., 2013; T. B. Monroe, Gore, Chen, et al., 2012; Scherder, Sergeant, and Swaab, 2003). As mentioned above, we have previously shown using the same methods described in this paper that people with a clinical diagnosis of AD demonstrated higher thermal thresholds for the detection of "warmth," "mild pain," and "moderate pain (T. B. Monroe, Gore, Bruehl, et al., 2015)." Others have found that people with AD demonstrated higher mechanical pressure pain thresholds and increased detection thresholds for electrical shock pain threshold (Cole et al., 2006; Gibson et al., 2001) respectively. Regarding sex effects on the detection of thermal pain stimuli unlike previous studies, the current study showed no difference between males and females in pain stimuli required to elicit the perception of pain. Interestingly, while controlling for covariates, the detection of thermal pain may be driven by possible depression (based on MMSE screening) in males.

Our results of the affective response to pain were like those reported for younger adults; males show greater pain-associated unpleasantness than females (Kunz, Scharmann, et al., 2007; Kunz, Mylius, et al., 2009). The current study found evidence to suggest that in males worsening global cognitive function was associated with higher reports of unpleasantness in the presence of weak and moderate pain. This finding supports Benedetti and colleagues (Benedetti et al., 1999), who reported increased tolerance to highly unpleasant evoked pain stimuli (electrical shock and ischemic arm pain) in people with worsening AD.

As an exploratory secondary analysis, there are some obvious limitations in the current study to consider when interpreting results. The parent study was not powered to detect sex differences, and the sample size was moderate. Other sensory modalities (e.g., somatosensory, auditory) may contribute to the overall pain experience which was not in the scope of this paper. As we have previously noted (T. B. Monroe, Gibson, et al., 2016), a perceptual matching paradigm was used rather than a fixed temperature for thermal sensory detection levels. This procedure may have impacted the pattern of findings for pain unpleasantness in unknown ways. To comply with IRB recommendation, we used 
the Method of Limits program on the Medoc Pathways Model so that the individual (regardless of cognition) was in control of the perceptual pain stopping point.

For this reason, we were unable to collect any perception above the rating of "moderate pain," specifically avoiding the perception of "severe pain" or the perception of "pain tolerance." This design was developed based on the mechanical pressure paradigm used by Cole and colleagues (Cole et al., 2006) in their perceptual matching paradigm of people with AD. Despite these limitations, the current study adds to the limited number of clinical and experimental pain studies examining sex-differences in altered pain response in people with cognitive impairment. Another strength of the design is that the authorship team includes authors with experience in the legal and ethical issues in pain research in people with cognitive impairment (T. B. Monroe, Herr, et al., 2013).

The clinical relevance of these findings suggests that, in either sex, the diminished ability to detect pain could lead to an increase in adverse outcomes, such as an increase in the length of hospitalization (Morrison et al., 2003). Additionally, the increased reporting of unpleasantness in males may increase the risk of suffering from pain in those with worsening cognition. Relative to cognitively healthy controls, people with worsening cognition generally have an altered response to clinical and experimental pain. Sex differences in pain reports compound the risk of increased pain in those with worsening cognition, especially when one-dimensional pain intensity scales are used that do not measure pain affect.

In summary, the current study found that sex-differences in pain experiences may be present in older adults with varying degrees of cognition. Future studies are needed to replicate these results and should include an examination of the response to fixed temperatures and neurophysiological basis of sex differences in pain processing in a wide range of ages and across the spectrum of cognitive functioning. In consideration of mounting evidence, we continue to advocate that clinicians consider using a multi-dimensional sensory and unpleasantness pain tool in clinical practice as males and females may ultimately report pain intensity and pain unpleasantness differently, regardless of cognition. Using pain intensity measures only may predispose to suffering by not capturing an essential dimension of pain. 


\section{Chapter 4}

\section{Sensory Changes as a Biomarker for Alzheimer's Disease $^{1}$}

\section{Background}

In the most recent publication of the "Alzheimer's Disease Facts and Figures" the Alzheimer's Association presented evidence suggesting a potential of savings of $\$ 7$ trillion dollars resulting from the early diagnosis of Alzheimer's disease (AD), a point at which $70 \%$ of those with $\mathrm{AD}$ are diagnosed in the prodromal phase of the illness or prior to the development of observable signs or symptoms of AD ("2019 Alzheimer's disease facts and figures" 2019). One current challenge is the lack of robustness in methods that detect AD, particularly in the very early stages of the disease. Early subtle changes in memory and cognition occur over the course of months to years making it difficult to recognize symptoms in patients, and often health care providers wrongly misattribute small changes in cognitive function as a process of normal aging (Knopman, Donohue, and Gutterman, 2000). Evidence of a complex pathophysiological process of AD is accumulating that indicates pathological change is detectable years before, rather than at the beginning of, the prodromal phase as neuropathologic examinations of adults clinically diagnosed without cognitive impairment reveal similar pathological signatures to those with AD (Dubois, Feldman, et al., 2007).

Recently, The National Institute on Aging (NIA) published a framework for the diagnosis of $\mathrm{AD}$ that shifted the understanding of $\mathrm{AD}$ as a syndrome or the clinical consequence of one or more diseases towards a biological process that incorporates measurable physiological parameters or biomarkers (Jack et al., 2018). Current biomarkers of AD are grouped into those of $\beta$ amyloid deposition (A), pathologic tau (T), and neurodegeneration $(\mathrm{N})$ and are typically gathered by advanced imaging and lumbar puncture (Jack et al.,

\footnotetext{
${ }^{1}$ Reprinted from the final submission with permission. Romano R.R., 3rd, M. A. Carter, and T. B. Monroe (2020). "Narrative Review of Sensory Changes as a Biomarker for Alzheimer's Disease". In:Biol Res Nurs,p. 1099800420947176. ISSN: 1552-4175 (Electronic) 1099-8004 (Linking)DOI:10.1177/1099800420947176. URL:http://www.ncbi.nlm.nih.gov/pubmed/32799655.
} 
2018). In the primary care setting it is difficult to detect pre-clinical AD because detection methods using imaging, invasive CSF test, and biomarker testing are rarely available outside of specialty clinics (Mortamais et al., 2017). Health care providers are advised to focus on the core criteria for diagnosis of clinical AD, which do not require biomarker evidence, but rely on clinical evaluation and neuropsychological testing (Tolea and Galvin, 2013). Since there is variation in the diagnostic abilities of primary care providers, research has focused on finding non-invasive biomarkers that may be helpful to health care providers in identifying AD (3. Romano R. R., Carter, Anderson, et al., 2020).

Alterations in sensations may be a useful biomarker. Senses are multisystem physiological mechanisms of perception traditionally categorized as smell, taste, sight, hearing, and touch, but also include the ability of humans to sense temperature (thermoception), body position (proprioception), pain (nociception), balance (equilibrioception), and touch, thirst and hunger (mechanoception) (Matlin and Foley, 1992). Stimuli interact with sensory receptors that initiate action potentials processed in the central nervous system to elicit an action (Matlin and Foley, 1992). In early AD, pathological changes in the brain may result in alteration in sensory perception and provide insight that may be helpful to identify those at risk for AD. This paper aims to explore what is known about current biomarkers using a focused and limited narrative review of the scientific literature pertaining to sensory changes as potential biomarkers for AD. Whittemore and Knafl, 2005 contend that a narrative review is the preferred method of organizing a body of literature, as this type of review allows for a combination of diverse methods to provide an in-depth understanding of a phenomena of interest and deepen understanding. The question posed for this review was "What is the current understanding of alterations in senses as potential biomarkers for AD?"

\section{Methods}

An analysis of the existing literature was undertaken to meet the study aim. Empirical studies published in English were included in this analysis. These included studies that examined traditional senses (sight, hearing, taste, smell, and touch); the detection of other stimuli (thermoception, proprioception, nociception, equilibrioception, and mechanoreception); and response to internal stimuli (hunger and thirst) that used multiple senses. Further, to be considered eligible, the sense had to be investigated in the study as a biomarker defined as a measurable and quantifiable biological parameter that can be used as an index for dementia. Data items extracted from the literature included method of sensation-testing, measure of clinical disease, and association with diagnosis.

A search of English-language studies reporting associations between alterations in sensory modalities and diagnosis of dementia was conducted using 3 databases: PubMed 
(May 2020), CINAHL (May 2020), and PsycINFO (May 2020). Search terms included "sensation," "dementia," "Alzheimer's disease," and "biomarker" as well as each sense individually (i.e., sight, hearing, taste, smell, touch, thermoception, proprioception, nociception, equilibrioception, and mechanoreception). All potential studies identified by this search $(\mathrm{N}=78)$ were exported directly to EndNote and reviewed for eligibility after duplicated studies were removed.

Articles were excluded if not topically related based on title and subsequent reading of the abstract to assure that a sense was investigated as a biomarker, defined as a measurable and quantifiable biological parameter used as an index for Alzheimer's disease. Further, articles identified as literature reviews, editorials, or commentaries were excluded, and manuscripts without readily available full texts were removed from the sample (see Figure 4.1). Studies were organized according to sensory systems. Data were extracted independently by the first author (RR) using a piloted data collection form, and any ambiguous information was settled by consensus among all authors. The quality of the studies was assessed using the study design-specific (e.g., observational cohort or crosssectional studies quality assessment tool) tool developed by the National Heart, Lung, and Blood Institute (NHLBI) and Research Triangle Institute International (Study Quality Assessment Tools, 2020). We awarded point values (e.g., No $=0$, Yes $=1$, Good $=2$, Fair $=1$, Poor $=0$ ) to each assessment of quality and totaled them for the final score quality score so that the higher the total score the better the quality.

\section{Results}

A total of 78 manuscripts were identified in the initial sample. The final literature review sample consisted of 18 studies after removing 3 duplicate manuscripts, 28 topically unrelated (e.g., ligand correlations to disease state, behavioral paradigms, depression related studies) or methodologically unacceptable manuscripts (e.g., reviews, commentaries, and editorials), 26 non-empirical manuscripts, and 3 non-human studies. The olfactory system was investigated in 12 manuscripts and was the most represented sense in the final sample. The other sensory systems in the final sample included auditory, vision, proprioception, and taste (Figure 4.1). No other sensory system studies were identified by this search.

\section{Olfactory}

Olfaction has been proposed as a biomarker for AD since Esiri and G. K. Wilcock, 1984 compared the olfactory bulbs in $\mathrm{AD}$ patients and normal controls and found that patients with AD had collections of neurofibrillary tangles in the anterior olfactory nucleus. There were 12 manuscripts reviewed concerning the olfactory sense as a biomarker for AD. All manuscripts were cross-sectional analyses, with 1 manuscript published in 1996 by Serby et al., 1996 having a 5-year follow-up (Table 4.1). 

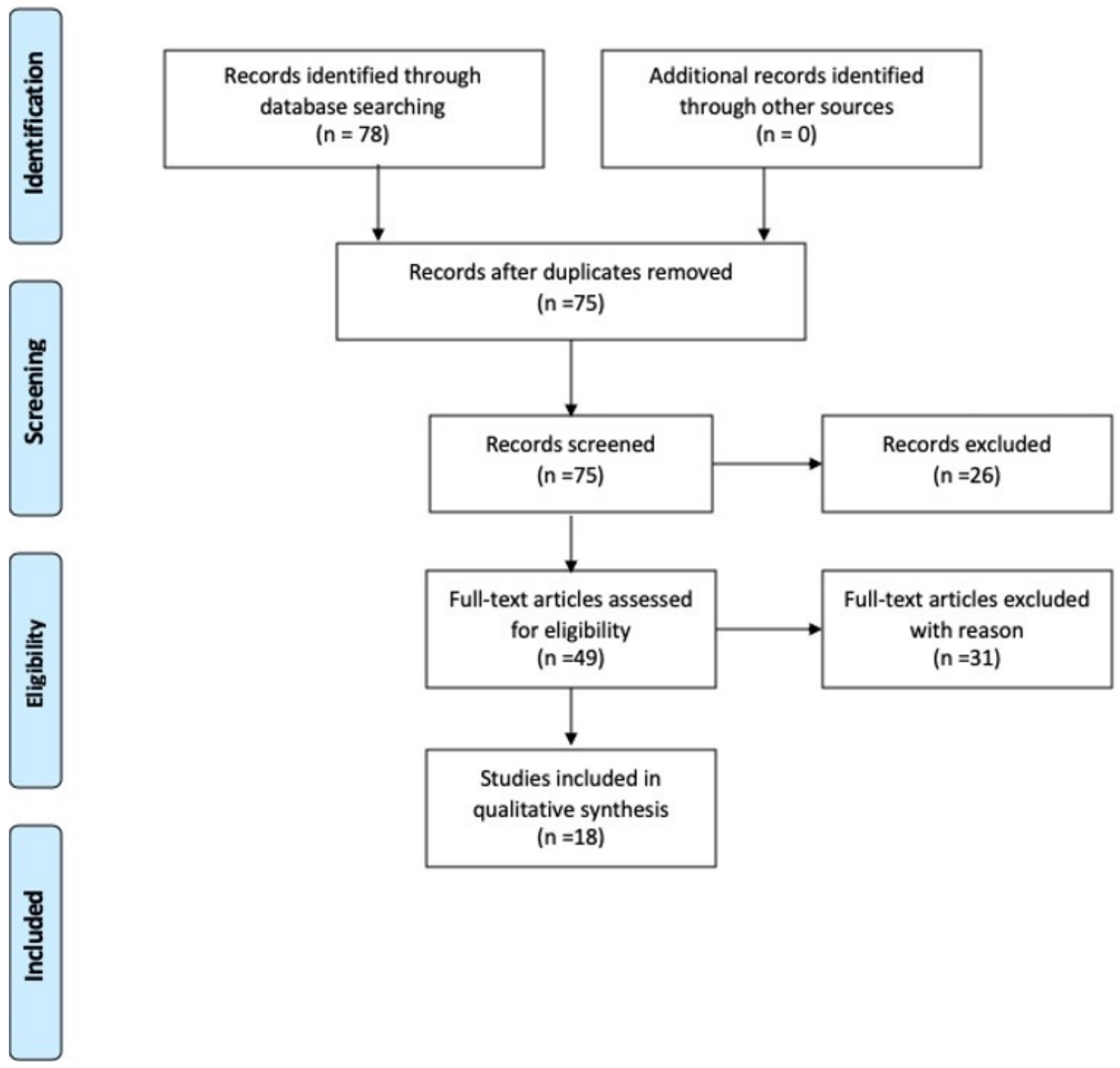

Figure 4.1: Consort prisma flow diagram. 
Table 4.1: Description of sample of 12 studies on olfaction as a biomarker.

\begin{tabular}{|c|c|c|c|c|c|}
\hline Author (Year) & Sample Size ${ }^{1}$ & Study Design & $\begin{array}{l}\text { Assessment of } \\
\text { Sensation }^{2}\end{array}$ & $\begin{array}{l}\text { Assessment of } \\
\text { Cognition }^{3}\end{array}$ & $\begin{array}{l}\text { Quality } \\
\text { Score }\end{array}$ \\
\hline Serby et al., 1996 & $\begin{array}{l}\mathrm{N}=28 \text { FDR } \\
\mathrm{N}=28 \text { controls }\end{array}$ & $\begin{array}{l}\text { Cross-sectional } \\
\text { 2-group comparison }\end{array}$ & UPSIT & MMSE, ADAS & 8 \\
\hline Wang et al., 2002 & $\begin{array}{l}\mathrm{N}=28 \mathrm{MCI} \\
\mathrm{N}=30 \text { controls }\end{array}$ & $\begin{array}{l}\text { Cross-sectional } \\
\text { 2-group } \\
\text { age-matched comparison }\end{array}$ & CC-SIT & CAMCOG-C, MMSE & 10 \\
\hline Peters et al., 2003 & $\begin{array}{l}\mathrm{N}=14 \mathrm{AD} \\
\mathrm{N}=8 \mathrm{MCI} \\
\mathrm{N}=8 \text { controls }\end{array}$ & $\begin{array}{l}\text { Cross-sectional } \\
\text { 3-group } \\
\text { age-matched comparison }\end{array}$ & $\begin{array}{l}\text { Butanol odor } \\
\text { odor discrimination } \\
\text { odor identification }\end{array}$ & MMSE, CDR & 10 \\
\hline $\begin{array}{l}\text { Olofsson } \\
\text { (2010) }\end{array}$ & $\mathrm{N}=1236$ & $\begin{array}{l}\text { 5-year longitudinal } \\
\text { cohort study }\end{array}$ & SOIT & MMSE, Vocabulary & 11 \\
\hline Jimbo et al., 2011 & $\begin{array}{l}\mathrm{N}=109 \text { AD } \\
\mathrm{N}=40 \text { controls }\end{array}$ & $\begin{array}{l}\text { Cross-sectional } \\
\text { 2-group } \\
\text { age-matched comparison }\end{array}$ & OSIT-J & MMSE, ADAS-cog & 9 \\
\hline Masaoka et al., 2013 & $\begin{array}{l}\mathrm{N}=16 \text { AD } \\
\mathrm{N}=\mathrm{PD} \\
\mathrm{N}=\mathrm{DM} 1 \\
\mathrm{~N}=67 \text { controls }\end{array}$ & $\begin{array}{l}\text { Cross-sectional } \\
\text { multi group comparison }\end{array}$ & UPSIT & $x$ & 10 \\
\hline Growdon et al., 2015 & $\mathrm{~N}=215 \mathrm{CN}$ & $\begin{array}{l}\text { Cross-sectional } \\
\text { 1-group analysis }\end{array}$ & UPSIT & MMSE, BNT-30, AmNART & 10 \\
\hline Dhilla Albers et al., 2016 & $\begin{array}{l}N=10 \mathrm{AD} \\
\mathrm{N}=29 \mathrm{MCI} \\
\mathrm{N}=74 \mathrm{SCC} \\
\mathrm{N}=70 \text { controls }\end{array}$ & $\begin{array}{l}\text { 4-group } \\
\text { cross-sectional analysis }\end{array}$ & OPID, POEM, OAS & CDR, MMSE, BNT, TrailsB & 9 \\
\hline Lafaille-Magnan et al., 2017 & $\mathrm{~N}=274$ & Cross-sectional & UPSIT & RBANS, MoCA & 11 \\
\hline Quarmley et al., 2017 & $\begin{array}{l}\mathrm{N}=262 \mathrm{AD} \\
\mathrm{N}=174 \mathrm{MCI} \\
\mathrm{N}=292 \text { controls }\end{array}$ & $\begin{array}{l}\text { Cross-sectional } \\
\text { 3-group comparison }\end{array}$ & SS-OIT & MoCA & 11 \\
\hline Tonacci et al., 2017 & $\begin{array}{l}\mathrm{N}=85 \mathrm{MCI} \\
\mathrm{N}=41 \text { controls }\end{array}$ & $\begin{array}{l}\text { Cross-sectional } \\
\text { age and gender-matched }\end{array}$ & OTT, ODT, OIT & $\begin{array}{l}\text { Free DCT, RAVLT, ROCF, BSS, } \\
\text { MMSE, CDR, ADL, ADAS-cog }\end{array}$ & 9 \\
\hline Kouzuki et al., 2018 & $\begin{array}{l}\mathrm{N}=40 \mathrm{AD} \\
\mathrm{N}=34 \mathrm{MCI} \\
\mathrm{N}=40 \text { controls }\end{array}$ & Cross-sectional analysis & OSIT-J & MMSE, ADAS-J, TDAS & 10 \\
\hline
\end{tabular}

${ }^{1}$ Abbreviations: FDR (First-degree relatives), MCI (Mild cognitive impairment), AD (Alzheimer's disease), PD (Parkinson Disease), DM1 (Diabetes Mellitus Type 1) SCC (Subjective Cognitive Concerns)

2 Abbreviations: UPSIT (University of Pennsylvania Smell Identification Test), CC-SIT(Cross-Cultural Smell Identification Test), SOIT (Scandinavian Odor IdentificationTest), OSIT-J (Odor Stick Identification Test for the Japanese), OPID (Odor PerceptIDentification), POEM (Perception of Odor Episodic Memory), OAS (Odor AwarenessScale), SS-OIT (Sniffin Sticks Odor Identification Test), OTT (Odor Threshold Testing, ODT (Odor Detection Threshold), OIT (Odor Identification Test).

${ }^{3}$ Abbreviations: MMSE (Mini Mental State Exam), ADAS (Alzheimer's Disease Assessment Scale), CAMCOG-C (Cambridge Cognitive Examination Chinese version), CDR (Clinical Dementia Rating), Vocabulary (Cureman and Salde, 1959), ADAS-cog (Alzheimer's Disease Assessment Scale-cognitive subscale), BNT (Boston Naming Test, AmNART (American National Adult Reading Test), TrailsB (Trails Making Test B), RBANS (Repeatable Battery for the Assessment of Neuropsychological Status), Free DCT (Dot Counting Test), RAVLT (Rey Auditory Verbal Learning Test), ROCF (Rey-Osterrieth complex figure test), BSS (Bhatia Battery of Performance tests of Intelligence), TDAS (Touch Panel-type Dementia Assessment Scale) 
The sample size of studies ranged from $n=30$ to $n=728$ and compared participants with $\mathrm{AD}$ to those with mild cognitive impairment, frontotemporal dementia, or healthy controls. Five studies were conducted in the United States, while the remaining studies were conducted in Japan $(n=2)$, Canada $(n=1)$, Sweden $(n=1)$, Italy $(n=1)$, Germany ( $=1)$, and China $(n=1)$. The quality of the manuscripts reviewed ranged from a score of 8-11, suggesting high-quality design and analysis.

Serby et al., 1996 found that first-degree relatives performed worse on a measure of odor identification when compared to a control group of community-dwelling older adults; further, the authors argued that impaired olfaction may be present before clinical symptoms. Similarly, 2 studies of participants with mild cognitive impairment (MCI), a known risk factor for $\mathrm{AD}$, showed odor identification was impaired in people with MCI when compared to age- and gender-matched, cognitively normal controls, further adding to the evidence that suggests changes in the olfactory system may be useful in identifying those at risk for AD (Peters et al., 2003; Tonacci et al., 2017). These subsequent studies used similar methods to Serby et al., 1996. The manuscripts in the sample all found significant associations with measures of olfaction and AD diagnosis (Quarmley et al., 2017). Two studies that specifically evaluated APOE4 status found significant associations with deficits in odor identification (Lafaille-Magnan et al., 2017; Wang et al., 2002). Most studies found olfactory function decreased in AD and MCI patients and was associated with either cerebral spinal fluid biomarker levels (Kouzuki et al., 2018; Lafaille-Magnan et al., 2017), genetic status (Lafaille-Magnan et al., 2017; Wang et al., 2002; Olofsson et al., 2010, worse performance on measures of cognitive function (Dhilla Albers et al., 2016; Jimbo et al., 2011; Lafaille-Magnan et al., 2017; Masaoka et al., 2013; Peters et al., 2003), or changes in brain structure as observed through imaging modalities (Growdon et al., 2015).

Though olfaction has been the most common sense evaluated as a potential biomarker for $\mathrm{AD}$, challenges may occur when using olfaction as a screening process in primary care because assuring reliability and specificity can be problematic since conditions such as seasonal allergies can yield confusing results. Cultural exposure to certain scents may vary and make it difficult to find a generalizable test. Offensive scents may also stimulate the trigeminal nerve rather than the olfactory nerve, further complicating the reliability of screening.

\section{Taste}

Taste and smell are closely related, as both senses converge in the caudal orbitofrontal cortex, yet research has mostly focused on the association between smell and AD. Neuroimaging techniques are able to visualize taste perception in cerebral representations and suggest that gustatory processing may be impaired in people with brain disease (Camicioli et al., 1998; Lang et al., 2006). Naudin et al., 2015 conducted a cross-sectional analysis and 
found that people with AD gave significantly more incorrect responses on salty, bitter, and sweet taste identification tasks when compared to healthy controls.

\section{Auditory}

The next most common sensory system reported as a potential biomarker has been hearing. The quality of the 2 studies included in this review can be considered strong, with scores of 10 on the design-specific quality assessment tool. Waldton, 1974 first showed functional impairment of the cranial nerves in 66 women with senile dementia. Further hearing loss becomes more prevalent as people age, making it a notable sense to study in the AD population. Autopsy studies have confirmed hallmarks of AD pathology, Aßplagues, and tau were identified in the cochlear nucleus and throughout the auditory system in patients with AD. Of the auditory manuscripts reviewed, found positive associations with changes in auditory response and either markers of $\mathrm{AD}$ (total-tau and p-tau) or measures of cognition (Laptinskaya et al., 2018; Tuwaig et al., 2017). The first study, published by Tuwaig et al., 2017, was a 2017 cross-sectional analysis of 187 cognitively normal, high-risk members of the PREVENT-AD cohort that found 2 tests of auditory abilities were associated with measures of cognition, concentration of total-tau and p-tau, measures of hippocampal and entorhinal cortical volumes, and cortical thickness. However, the authors did not find an association between the measures of central auditory processing and APOE4 carrier status.

The second auditory study reported significant changes in the event-related response in an EEG signal caused by alteration of auditory stimulation after a long interstimulus interval, and this change in EEG signal was associated with worse episodic memory performance at five-year follow-up (Laptinskaya et al., 2018). Although limited, there is growing evidence of hearing loss as a potential indicator of AD; however, it is unclear if these changes are associated with AD pathology or with temporal lobe abnormalities related to aging (Murphy, 2019).

\section{Vision}

Changes in vision has been identified as another possible marker of $\mathrm{AD}$, given that amyloidbeta plagues have been identified in the aqueous humorous of AD patients undergoing cataract surgery. In 2013, Risacher and colleagues accurately identified people with MCI relative to healthy controls using vision testing (Risacher, Wudunn, et al., 2013). The authors also determined that people with $\mathrm{AD}$ and $\mathrm{MCI}$ demonstrated significant contrast sensitivity visual deficits, which also is associated with worse performance of cognitive measures.

In a later cross-sectional analysis, Balachandar and colleagues (2017) found that people with mild AD exhibited significant associations between deficits on visuospatial measures and changes in resting-state functional connectivity (Balachandar et al., 2017). 
Vision changes may prove to be an inadequate biomarker, as there remains discussion on the timing of vision pathology and AD clinical disease as well as the fact that alteration in vision is a part of healthy aging in addition to part of other confounding disease processes (Murphy, 2019).

\section{Proprioception}

Proprioception, or the awareness and perception of one's body in space, has the potential to be an early marker of AD pathology, as motor decline is observed in all stages of AD (Boyle et al., 2007). A slowing of walking speed that proceeds cognitive decline was first observed 2 decades ago and is explained by the overlap in the brain structures (i. e., the cerebellum, basal ganglia, and motor cortex) responsible for walking and involved in attentional, executive, and visuospatial functions (Camicioli et al., 1998). Tu and colleagues (2015) used a virtual supermarket task to discriminate between participants with AD and frontotemporal dementia (Tu et al., 2015). The authors found that people with AD had more impairment in orientation compared to people with FTD and concluded that orientation had the same level of diagnostic sensitivity as other measures of episodic memory (Tu et al., 2015). This evidence supports the use of tests of proprioception as a tool to identify persons with AD.

\section{Discussion}

The current state of the literature shows a paucity of research in how non-traditional senses may be used as biomarkers for AD. Yet, the results do suggest further research is warranted as the majority of studies reviewed demonstrate that sense perception changes at different diagnosis categories (e.g., AD, MCI, normal). Results from this review are promising as they suggest that future research in this area could shed light on other methods of assessment that may be more feasible and less invasive than current practice recommendations such as lumbar puncture, MRI, and PET scans to identify those with Alzheimer's disease. Overall, olfaction was the most widely studied sense. Researchers of the 12 manuscripts reviewed showed measures of olfaction were associated with changes in cognition, imaging, and APOE status. Though limited in scope, this review demonstrates how a lack of evidence supporting how other senses could be used as a biomarker for AD serves as a clear gap in the literature. Evidence from all manuscripts included in the current review indicates deficits or changes in senses can potentially be used to differentiate between people with AD, MCI, and cognitively normal adults.

One possible, yet under studied area for AD biomarkers is pain. AD is well-known to cause changes in brain structures and metabolism (T. B. Monroe, Beach, et al., 2017; T. B. Monroe, Fillingim, et al., 2018). The hippocampus and prefrontal cortex experience the most brain volume loss with healthy aging; however, in individuals with $\mathrm{AD}$, the entorhinal cortex and hippocampus and the lateral temporal lobe and neocortex have significantly 
more volume loss compared to healthy individuals (T. B. Monroe, Gore, Chen, et al., 2012). As $\mathrm{AD}$ progresses, damage typically occurs in both the lateral and medial pain network. Studies have shown that neurofibrillary tangles and $\beta$ amyloid plaques accumulate in the amygdala (Kromer Vogt et al., 1990), orbitofrontal cortex (Tekin et al., 2001), insula, PAG (Parvizi, Van Hoesen, and Damasio, 2000), and striatum (Selden et al., 1994), all of which comprise the rostral pain system (T. B. Monroe, Gore, Chen, et al., 2012). People with AD have alterations in pain perceptions, and this has been shown to be different when compared to cognitively normal adults (T. B. Monroe, Gibson, et al., 2016; T. B. Monroe, Gore, Bruehl, et al., 2015; R. R. Romano et al., 2019). Measuring pain perception in individuals who are very early in the process of $\mathrm{AD}$ is not complex and could be adapted to most primary care practices. Also important is that the association between changes in pain perception in AD is theoretically sound (T. B. Monroe, Gore, Chen, et al., 2012). Pain is not a single sense per se, as multiple areas of the brain are used in pain perception, and this complexity may allow alterations in pain perception to be used as an early biomarker for AD.

\section{Implications for Research}

New therapeutic agents are being explored that hold promise for clinicians to be able to offer treatment that alters the progression of $\mathrm{AD}$ (Cummings et al., 2019). However, the state of the science in identifying non-invasive biomarkers for $\mathrm{AD}$ that can be identified prior to the onset of cognitive impairment is still in the early stages. The results of this review suggest that additional focus should be directed on alternation of senses as a potentially useful biomarker. Further the results of this review identify a clear gap in the literature as only one nontraditional sense manuscript was identified through this review. If senses prove to be useful noninvasive biomarkers then clinicians might have feasible, simple, and non-invasive methods available to identify people who have early or asymptomatic AD for early treatment.

\section{Limitations}

Narrative reviews have a number of potential limitations. Defined parameters and boundaries can pose a challenge. In this study, the selection of the search terms was believed to be appropriate, but there is a possibility that some work may have been missed, as this is a rarely explored field of scientific work. Every effort was made to assure that the widest search of the actual research literature was conducted, but the language and/or MeSH terms may still be ambiguous. Further, the majority of data collection was conducted by the primary author; however, the final sample of manuscripts were reviewed by all authors and any disagreement on interpretation of the results (Table 4.2) were discussed and interpreted by group consensus. 
Table 4.2: Quality scores of auditory, vision, proprioception, and taste studies.

\begin{tabular}{lc}
\hline Author (Year) & Quality Score $^{1}$ \\
\hline Laptinskaya (2018) & 10 \\
Tuwaig (2017) & 10 \\
Balachandar (2017) & 9 \\
Risacher (2013) & 9 \\
Tu (2015) & 9 \\
Naudin (2015) & 10 \\
\hline 1 Study Quality & Assessment Tools. \\
(2020). National Heart, Lung, \\
and Blood Institute. $\quad$ Retrieved \\
July 13 2020 from https://www. \\
nhlbi.nih.gov/health-topics/ \\
study-quality-assessment-tools
\end{tabular}




\section{Chapter 5}

\section{Could an Altered Evoked Pain Response Be a Phenotypic Biomarker for Alzheimer's Disease Risk? ${ }^{1}$}

\section{Background}

Earlier diagnosis of Alzheimer's disease (AD) has been shown to reduce cost and improve patient outcomes despite the limited availability of current treatment options ("2020 Alzheimer's disease facts and figures" 2020). One method used to initiate earlier diagnosis is identifying AD prior to the onset of classical signs or symptoms, and biomarkers hold potential of serving this function. Currently recognized biomarkers of AD are invasive and not practical in the primary care setting where the majority of patients with memory concerns first seek care ("2020 Alzheimer's disease facts and figures" 2020; Wilkinson et al., 2004. An initial report of increased neurofibrillary tangles in olfactory bulb in people with AD spurred further studies directly investigating sensory processing in AD. (Esiri and G. K. Wilcock, 1984). Since that early report, studies have demonstrated that changes in olfaction, taste, hearing, vision, and proprioception have the potential to serve as biomarkers of AD (3. Romano R. R., Carter, and T. B. Monroe, 2020).

Current approaches used to identify individuals with AD include lumbar puncture with analysis of cerebral spinal fluid, magnetic resonance imaging, and positron emission tomography scans ("2020 Alzheimer's disease facts and figures" 2020). These approaches are invasive, costly, and not widely available or feasible in the primary care setting(Iliffe, Robinson, et al., 2009). In contrast, measures of sensory changes are less invasive, more feasible, and readily available in primary care practices where most individuals present for initial diagnosis (Wilkinson et al., 2004; 3. Romano R. R., Carter, and T. B. Monroe, 2020). Sensory processing for all senses (e.g., olfaction, vision, proprioception) involves multisystem physiological mechanisms in which stimuli activate sensory receptors, thereby initiating action potentials leading to central nervous system (CNS) processing and ultimately a

\footnotetext{
${ }^{1}$ Romano R. R., 3rd, M. A. Carter, M. S. Dietrich, R. L. Cowan, S. P. Bruehl and T. B. Monroe. "Could Altered Evoked Pain Responsiveness Be a Phenotypic Biomarker for Alzheimer's Disease Risk?: A Cross-Sectional Analysis of Cognitively Healthy Individuals." Journal of Alzheimer's Disease. In press.
} 
perceptual response(Matlin and Foley, 1992).AD-related changes in the CNS have the potential to alter all such sensory processing (3. Romano R. R., Carter, and T. B. Monroe, 2020). Processing of pain stimuli in the CNS occurs in the lateral (sensory) and medial (emotional) brain networks with the integration of the rostral (behavioral) network in healthy individuals and at varying degrees of neurocognitive impairment, people may present with increased or decreased pain-related behaviors coupled with increased or decreased reports of pain intensity and pain unpleaseantness (T. B. Monroe, Gore, Chen, et al., 2012). Several studies have demonstrated that the perception of controlled experimentally-induced pain stimuli differs between cognitively healthy people and people with AD (T. B. Monroe, Gore, Chen, et al., 2012; T. B. Monroe, Beach, et al., 2017; T. B. Monroe, Fillingim, et al., 2018; T. B. Monroe, Gibson, et al., 2016; R. R. Romano et al., 2019, which suggests that pain processing is altered in the AD. However, changes in pain processing have not been directly linked to genetic markers for AD. Thus to date, the phenotypic association of the $\varepsilon 4$ allele has not been considered in pain outcomes.

The $\varepsilon 4$ allele of apolipoprotein E (APOE) is a genetic marker associated with an increased incidence of developing late-onset $\mathrm{AD}$ and is a risk factor identifiable long before the onset of AD symptoms (Najm, E. A. Jones, and Huang, 2019; Safieh, Korczyn, and Michaelson, 2019; Yamazaki et al., 2019). The APOE gene encodes the production of a 299 amino acid ligand that is primarily synthesized in astrocytes of glial cells in the brain, the liver, and macrophages in peripheral tissue and is mostly responsible for transporting cholesterol (Najm, E. A. Jones, and Huang, 2019; Safieh, Korczyn, and Michaelson, 2019; Yamazaki et al., 2019; Maehlen et al., 2013). APOE can take on three isoforms dictated by three polymorphic alleles- $\varepsilon 2, \varepsilon 3$, and $\varepsilon 4$-resulting from a difference in only one or two amino acids (Safieh, Korczyn, and Michaelson, 2019; Yamazaki et al., 2019). The most common allele, APOE $\varepsilon 3$, is present in $50-90 \%$ of all populations; APOE $\varepsilon 4$ is distributed in about 5-35\%; and APOE $\varepsilon 2$, the least common allele, is distrubted in about $1-5 \%$ of the population (Mahley and Rall, 2000; Verghese, Castellano, and Holtzman, 2011; Ward et al., 2012). Genetically, a person can be homozygous (i.e., $\varepsilon 4 / \varepsilon 4, \varepsilon 3 / \varepsilon 3$, and $\varepsilon 2 / \varepsilon 2$ ) for one major subtype or heterozygous $(\varepsilon 4 / \varepsilon 3, \varepsilon 3 / \varepsilon 2$, and $\varepsilon 4 / \varepsilon 2)$ for two main subtypes, resulting in six different phenotypic populations (Yamazaki et al., 2019). The risk of developing $\mathrm{AD}$ changes between genetic profiles, as those with the greatest odds of developing AD include $\varepsilon 2 / \varepsilon 4, \varepsilon 3 / \varepsilon 4$, and $\varepsilon 4 / \varepsilon 4$, while those with reduced odds include $\varepsilon 2 / \varepsilon 2$ and $\varepsilon 2 / \varepsilon 3$ (Najm, E. A. Jones, and Huang, 2019; Yamazaki et al., 2019; Maehlen et al., 2013). A recent meta analysis of AD populations estimated about $49 \%$ as being APOE $\varepsilon 4$ carriers and about $10 \%$ as $\varepsilon 4 / \varepsilon 4$ positive carriers and noted that carrying at least one copy of the $\varepsilon 4$ allele increases risk 3-fold when compared to healthy controls and almost 12-fold for those with two $\varepsilon 4$ alleles and When compared to $\varepsilon 3$ carriers, APOE $\varepsilon 4$ positive individuals have three times the risk of developing AD (Ward et al., 2012).

The aim of the current study was to determine whether individuals at increased risk of late-onset $\mathrm{AD}$ based on $\mathrm{APOE}$ allele genotype differ phenotypically in their response to 
experimentally-induced painful stimuli compared to those who do not have at least one copy of $\varepsilon 4$ allele.

\section{Methods}

\section{Study Participants}

Recruitment, enrollment, psychophysical testing, and cognitive assessments techniques are previously described (T. B. Monroe, Beach, et al., 2017). English-speaking, verbally communicative participants aged 30-89 years were recruited between 2014 and 2017 from a Mid-south metropolitan area. Participants were excluded for the presence of chronic pain; cognitive impairment (Mini-Mental State Exam [MMSE] < 28) (M. F. Folstein, S. E. Folstein, and McHugh, 1975); daily use of analgesic medication; or history of stroke, cancer, peripheral neuropathy, Raynaud's Disease, unstable medical conditions (e.g., severe restrictive or obstructive lung disease), insulin-dependent diabetes, current substance use disorders, or psychiatric diagnoses of bipolar disorder, major depressive disorder, schizophrenia; or presence of movement disorders including Parkinson's disease and restless leg syndrome. The Vanderbilt University Institutional Review Board approved the study. All participants were compensated US \$100 for their time.

\section{Screening and Enrollment}

Eligibility was assessed using a two-part screening process via telephone or in-person visit. Participants underwent one hour of psychosocial assessment, including medication use, demographic information, Hollingshead Four-Factor SES (Hollingshead, 1975) and cognitive screening with the MMSE (M. F. Folstein, S. E. Folstein, and McHugh, 1975). Participants were assessed with the Brief Pain Inventory (BPI) (Cleeland and Ryan, 1994), 5-item World Health Organization Well-Being Index (Hamilton, 1960), and State-Trait Anxiety Inventory (STAI) (Spielberger, 1971).

\section{Thermal Stimulation Protocol (Psychophysics)}

The thermal stimulation protocol used in the study assessed two aspects of pain: the intensity of pain and the unpleasantness of pain. The protocol was a modification of the perceptual matching experimental mechanical pressure pain protocol used by Cole and colleagues (Cole et al., 2006) and used the Medoc Q-Sense ${ }^{\mathrm{TM}}$. This device evokes simulation of A-delta and C-fibers (Hunt and Koltzenburg, 2005; Wager et al., 2004). The Medoc thermode $(30 \times 30 \mathrm{~mm})$ was attached to the thenar eminence of the right hand of each participant, and participants were shown a 0-20 sensory pain intensity scale and asked to stop the heat stimulus (via clicking a computer mouse) when they felt "just noticeable pain," "weak pain," or "moderate pain" (with each precept tested in separate trials). Participants were then asked to rate the unpleasantness of the sensation at each pain intensity percept 
using a 0-20 unpleasantness scale with the following anchors: " $0=$ neutral," " $5=$ slightly unpleasant," " $8=$ unpleasant," "11=very unpleasant," "16=intolerable," and "20=extremely distressing"(Petzke et al., 2005).

The baseline temperature was set as $30^{\circ} \mathrm{C}$ (a temperature not perceived as warm or cold), and the thermode was programmed to deliver heat that increased at a rate of $4^{\circ} \mathrm{C} /$ second. We modeled our thermal stimulus delivery after Wager and colleagues' paradigm in which each temperature stimulus began from baseline and ramped up and down at a moderate rate (Wager et al., 2004). Subsequently, we recorded the temperature at which each participant reported the perceptions of just noticeable pain (indicator of pain threshold), weak pain, and moderate pain. Each participant completed three pseudorandomized trials consisting of two instances at each percept level. The average temperature $\left({ }^{\circ} \mathrm{C}\right)$ across the trials at each level of intensity was used in analyses (maximum temperature $=50^{\circ} \mathrm{C}$ ). Immediately after indicating the first stimulus meeting criteria for each of the three intensity levels, participants were asked to rate the unpleasantness associated with that stimulus level as described above. Ten seconds of rest was provided between each percept in each trial.

\section{APOE4 Status Determination}

Participants supplied $2 \mathrm{~mL}$ of saliva which was collected and stored in an Oragene $\mathrm{TM}^{\mathrm{TM}}$ saliva collection tube. APOE genotype was determined using TaqMan ${ }^{\mathrm{TM}}$ assays. For statistical analysis, participants were categorized as either being APOE4 positive (APOE $\varepsilon 3 / \varepsilon 4$, $\varepsilon 4 / \varepsilon 4$ ) or APOE4 negative (APOE $\varepsilon 2 / \varepsilon 2, \varepsilon 2 / \varepsilon 3, \varepsilon 3 / \varepsilon 3$ ).

\section{Statistical Analysis}

Frequency distributions were used to summarize nominal and ordinal data. Due to the skewness of many of the continuous distributions, median and inter-quartile range were used to summarize the continuously scaled measures. Mixed-effects (between-subject: APOE4 allele status; within-subject: three percept levels) general linear models tested the main and interaction effect of allele status (negative, positive) and percept level (warmth, mild pain, moderate pain) on the temperature at which each level was reported and on the unpleasantness value of the pain at that level. Pairwise Mann-Whitney $U$ tests were used to assess pairwise differences at each percept level. Distributions were square root transformed to meet the normal assumptions of the models used and to calculate Cohen's $\mathrm{d}$ effect size statistics for the group differences at each threshold level. An alpha level of $\mathrm{p}<0.05$, set a priori, was used for statistical significance determinations. 


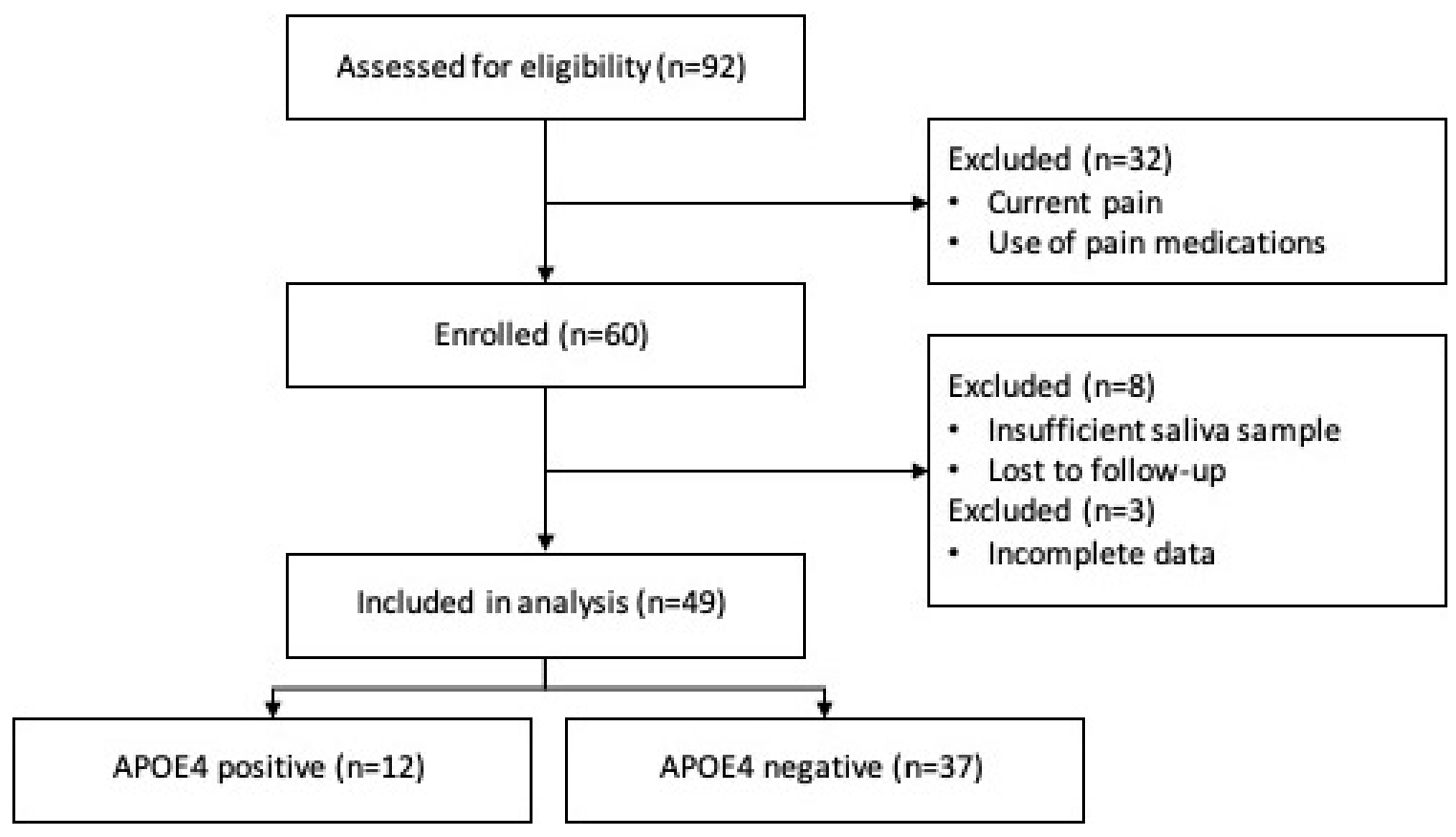

Figure 5.1: Consort flow diagram.

\section{Results}

\section{Sample Characteristics}

A total of 92 participants were screened; 32 participants were excluded prior to enrollment for not meeting inclusion criteria; 60 participants were enrolled; of those, 1 participant was lost to follow-up, 7 participants were excluded for failure to provide sufficient salvia volume for genetic analysis, and 3 participants had incomplete data leaving a final sample of 49 (Figure 5.1).The majority of participants carried the $\varepsilon 3 / \varepsilon 3$ alleles $(n=27,55 \%)$, and the remaining allele distributions were $\varepsilon 3 / \varepsilon 4(\mathrm{n}=10,20 \%), \varepsilon 2 / \varepsilon 3(\mathrm{n}=8,16 \%), \varepsilon 2 / \varepsilon 2(\mathrm{n}=2$, $4 \%)$, and $\varepsilon 4 / \varepsilon 4(n=2,4 \%)$. There were no participants with $\varepsilon 2 / \varepsilon 4$ alleles. Slightly more than half the sample was male $(n=26,53 \%)$ and a majority were white $(n=38,78 \%)$. Sample characteristics are summarized in Table 5.1. Median age of the sample was 68.0 years (IQR: 48-80 and median MMSE score was 30.0 (IQR: 29-30). There were no statistically significant differences between APOE4 positive $(n=12)$ and APOE4 negative $(n=37)$ participants on any of the characteristics investigated including average pain and current pain scores on the BPI $(p>.05$, Table 5.1). 
Table 5.1: Demographic and sample characteristics.

\begin{tabular}{lcccc}
\hline Psychophysics & $\begin{array}{c}\text { APOE4 Negative } \\
(\mathbf{n = 3 7 )}\end{array}$ & $\begin{array}{c}\text { APOE4 Positive } \\
(\mathbf{n = 1 2})\end{array}$ & $\begin{array}{c}\text { Total } \\
(\mathbf{n = 4 9 )}\end{array}$ & P-value \\
\hline & & Median (IQR) & & \\
Age & $68.0(48-80)$ & $49.0(33-80)$ & $65.0(46-80)$ & .192 \\
SES $^{1}$ & $52.5(49-58)$ & $58.0(51-88)$ & $53.5(49-60)$ & .131 \\
MMSE $^{2}$ & $30.0(29-30)$ & $30(29-30)$ & $30(29-30)$ & .339 \\
BPI-SF average pain $^{3}$ & $0(0-0)$ & $0(0-0)$ & $0(0-0)$ & .783 \\
BPI-SF pain right now $^{3}$ & $0(0-0)$ & $0(0-0)$ & $0(0-0)$ & .817 \\
WHO-5 $^{4}$ & $19.0(17-21)$ & $19.5(16-21)$ & $19.0(17-21)$ & .699 \\
STAI state Y form $^{5}$ & $15.0(15-16)$ & $15.0(14-15)$ & $15.0(15-15)$ & .544 \\
STAI trait $^{6}$ & $47.0(45-49)$ & $46.5(44-49)$ & $47.0(44-49)$ & .565 \\
\hline
\end{tabular}

${ }^{1}$ Hollingshead Four-Factor Measure of Socioeconomic Status (range=8-66; 8=lowest SES, 66=highest SES); $\mathrm{N}=40$, Negative $=31$, Positive $=9$

2 MMSE-Folstein Mini-Mental State Examination (range $=0-30 ; 0=$ completely cognitively impaired, $30=$ completely cognitively healthy)

${ }^{3}$ BPI-SF-Brief Pain Inventory Short Form (range=0-10; $0=$ no pain, $10=$ most pain); Current Pain $\mathrm{N}=48$, Negative $=37$, Positive $=11$

${ }^{4}$ WHO-5 Well-Being Index (range=0-25, 25=maximal well-being)

${ }^{5}$ STAI-Spielberger State Anxiety Inventory-STATE Y form (range 6-24; 6=increased anxiety, 24=least amount of anxiety)

${ }^{6}$ STAI-Spielberger Trait Anxiety Inventory (range=20-80; 20=increased anxiety, $80=$ least amount of anxiety); $\mathrm{N}=48$, Negative $=36$, Positive $=12$ 
Chapter 5. Could an Altered Evoked Pain Response Be a Phenotypic Biomarker for Alzheimer's Disease Risk?

Table 5.2: Experience of pain between APOE4 negative and positive participants.

\begin{tabular}{|c|c|c|c|c|}
\hline Psychophysics & $\begin{array}{c}\text { APOE4 Negative } \\
(n=37)\end{array}$ & $\begin{array}{l}\text { APOE4 Positive } \\
(n=12)\end{array}$ & p-value ${ }^{1}$ & Cohen's d \\
\hline Temperature & \multicolumn{2}{|c|}{ Median ${ }^{\circ} \mathrm{C}$ (IQR) } & & \\
\hline Overall & $40.0(35-45)$ & $42.0(35-48)$ & .040 & \\
\hline $\begin{array}{l}\text { Just noticeable } \\
\text { warmth }\end{array}$ & $34.0(32-37)$ & $34.1(32-36)$ & .806 & 0.16 \\
\hline Weak pain & $41.0(37-43)$ & $42.5(39-46)$ & .139 & 0.54 \\
\hline Moderate pain & $45.3(43-48)$ & $47.9(45-49)$ & .057 & 0.59 \\
\hline Unpleasantness & \multicolumn{2}{|c|}{ Median (IQR) } & & \\
\hline Overall & $4.0(0-7)$ & $5.5(1-10)$ & .014 & \\
\hline $\begin{array}{l}\text { Just noticeable } \\
\text { warmth }\end{array}$ & $0.0(0-1)$ & $0.2(0-2)$ & .395 & 0.23 \\
\hline Weak pain & $4.0(1-6)$ & $5.8(4-8)$ & .060 & 0.52 \\
\hline Moderate pain & $8.0(6-10)$ & $10.0(8-13)$ & .080 & 0.50 \\
\hline
\end{tabular}

\section{APOE and Pain}

The psychophysical data for APOE4 positive and APOE4 negative participants are summarized in Table 5.2.

Statistically significant main effects of increasing percept intensity were observed on both stimulus temperature and unpleasantness $(\mathrm{p}<.001)$. Thus, as expected, stimulus temperature and unpleasantness both increased as the targeted percept intensity increased from just noticeable to moderate pain. Our primary focus was on the main effects of APOE4 status. Statistically significant main effects of APOE4 status were noted for the temperature necessary to elicit the three targeted pain percepts $(p=.040)$ and on the experience of unpleasantness of that pain $(\mathrm{p}=.014)$. The APOE4 positive participants were less pain sensitive overall, that is, they reported reaching the targeted pain percepts at a significantly higher temperature than did those who were APOE4 negative. However, when those pain percepts were reached, APOE4 positive participants reported that pain to be more unpleasant relative to APOE4 negative participants. As shown in Table 5.2, the 
interaction effects of APOE4 status and pain percept level in both models were not statistically significant ( $p>.05)$, yet as is apparent, the strongest effects of APOE4 status were observed at the weak and moderate pain levels (Cohen's $d=0.50$ to 0.59). A follow-up analysis examining the effects of APOE4 on pain unpleasantness adjusting for differences in stimulus intensity at each percept level across genetic groups indicated that the pattern of results was little changed, with the APOE4 main effect becoming marginally significant $(p<.09)$ but the overall effect size increasing slightly (from $d=0.26$ to $d=0.33$ ).

\section{Discussion}

In this study, we found that cognitively healthy APOE4 positive individuals who are at increased genetic risk of late-onset AD exhibit significantly reduced sensitivity to evoked thermal pain relative to APOE4 negative individuals. However, when specific pain percepts are reached, APOE4 positive individuals report this pain to be more unpleasant than individuals without an APOE4 allele. To the best of our knowledge, this is the first report of an association of APOE4 allele status with an altered response to pain in a cognitively healthy sample of adults across the life span. Notably, the observed psychophysical results in this work mirror a recent study in which the authors used a very similar study design to test pain perception differences between cognitively healthy adults and people with diagnosed AD age 65 and older (T. B. Monroe, Gibson, et al., 2016). As in the current work, people with AD required a higher stimulus temperature than cognitively healthy controls to report the perceptions of "warmth" "mild pain" and "moderate pain". However, APOE status was not available for analysis in that prior work. In context of this prioir work, these present results suggest that thermal evoked pain testing could serve as a potential phenotypic biomarker of individuals at increased risk for AD. At minimum, further research on this issue appears to be warranted in a larger sample. Differences observed between APOE4 positive and APOE4 negative cognitively normal individuals in thermal percept detection levels and in pain unpleaseantness at each percept may hint at possible mechanisms for how prodromal AD pathology may disrupt pain processing (T. B. Monroe, Beach, et al., 2017; T. B. Monroe, Fillingim, et al., 2018; T. B. Monroe, Gibson, et al., 2016; R. R. Romano et al., 2019). This possibility is consistent with other work suggesting that alterations in other sensory systems are potential phenotypic markers for subsequent AD risk (3. Romano R. R., Carter, and T. B. Monroe, 2020).

This study does have some limitations. First, the use of a perceptual matching paradigm in which participants reported unpleasantness of pain at temperature intensities unique to each individual potentially leads to confounded assessmment for this outcome. In future work, this potential counfound can be overcome through using a series of fixed temperatures across participants. Another limitation is the small sample size, which reduced statistical power and likely adversely impacted ability to test the effect of APOE4 at each percept level individually. Despite this limitation, the mixed effect models 
did demonstrate statistically significant overall differences as a function of APOE4 status across the three percept levels. Moreover, the moderate effect sizes for between group differences noted at the mild pain and moderate pain percept levels suggest that APOE4related differences in pain perception that are likely to be clinically meaningful. Future studies should aim to replicate the current results in larger samples and with other types of experimental pain stimuli, such as a mechanical pressure pain or ischemic pain.

As the prevalence of dementia is expected to triple over the next 30 years, more evidence is needed to understand the altered pain experience in people with AD ("2020 Alzheimer's disease facts and figures" 2020; Johannes et al., 2010). Carriers of the $\varepsilon 4$ allele are at greater risk for amloyid deposition, impacting brain structures such as the hypothalamus and the prefrontal cortex, which may disrupt neural circuits mediating pain perception and behavioral expression that result in differences in psychophysical measurements of pain (Cole et al., 2006; Scherder, Sergeant, and Swaab, 2003; Stubbs et al., 2016).The results of this research demonstrate for the first time that alterations in evoked pain responsiveness may be a potential phenotypic marker for identifying those at risk for APOE4-related late-onset AD (3. Romano R. R., Carter, and T. B. Monroe, 2020). In the clinical context, the possibility that APOE4 allele status may alter the risk of pain-related suffering (either by directly increasing pain unpleasantness or delaying necessary medical care due to decreased pain sensitivity) irrespective of AD status may warrant further exploration. 


\section{Chapter 6}

\section{Discussion}

\section{Summary}

The aim of this research was to find ways for primary care providers to recognize AD early enough in the preclinical stage to affect outcomes of care. Chapter 2 presented the first published manuscript, an integrative review, highlighting the complexity of barriers that exist at each level of the health care system that contribute to limit diagnoses of AD by PCPs. One study reviewed suggested that the comorbitities or complexity of the patients' presenting problems makes it difficult to diagnose AD. Pain is a common cormorbidity seen in $\mathrm{AD}$ and a main reason people interact with the health care system. This funding led to the second published study. The second study was undertaken to explore how pain may differ in those at risk for AD. Chapter 3 presented the results of a study demonstrating that the experience of pain differs between males and females as a measure of cognition worsens suggesting pain may be a useful marker of AD. This led to the third published study. Chapter 4 presented a narrative review of the literature demonstrating that differences in senses have been associated with the different diagnostic categories and that there is a paucity of research in how pain may be used as a marker of AD. This conclusion formed the basis of the fourth study. Chapter 5 reported an analysis of data in a study that included APOE4 association with pain response in cognitively healthy individuals. This compared individuals at increased risk of AD experience pain based on their APOE4 status at a greater temperature and found that pain was more unpleasant when compared to those without the increased risk of AD. We believe that this is the first time this finding has been reported. Further work is warranted to understand how the experience of pain maybe used as a biomarker tool to identify those who are susceptible to subsequent development of AD.

\section{Limitations}

A major limitation for the study that tested associations of APOE4 status and pain was the small sample size. Future work should focus on enrolling more participants with a greater 
proportion of APOE4 positive individuals and exposing them to different types of experimental pain over a period of time. The results would also be stronger if a younger cohort was followed and additional biomarkers were collected (e.g., MRI, CSF tau) to explore if differences in the experience of pain is also different between those positive or negative for markers of $\mathrm{T}$ or $(\mathrm{N})$. Exploratory genetic markers could be collected, such as polymorphisms of the gene for cyclin-dependent kinase 5 (CDK5) which has been associated with neuropathic pain and APOE. Further the differences in the specific genetic profiles can be teased out with more subjects but may be too difficult to reach enrollment needs based on the current distribution to clinical studies.

\section{Recommendations for Future Directions}

The search to identify preclinical markers of AD would provide clues to later onset of $A D$ in individuals at risk. This would allow for earlier interventions and the potential use of disease modifying medications once approved. Older adults and their loved ones often seek care from their primary care providers with initial concerns for memory. Their primary care providers have been identified as key to improve diagnosis which is important because as we currently stand interventions aimed at PCPS to improve diagnosis are not having the desired effect. There is evidence that primary care providers' diagnostic accuracy is poor and it takes about 17 years for research to catch up to in clinical practice ("2020 Alzheimer's disease facts and figures" 2020). We need to identify earlier markers of Alzheimer's disease. The evidence of this research suggests pain as a possible early marker of AD.

The evidence presented in this dissertation complements previous findings suggesting people at risk for AD based on carrying the $\varepsilon 4$ allele had significantly great brain activation in multiple regions involved in pain processing when compared to those without the $\varepsilon 4$ allele (T. B. Monroe, Gore, Chen, et al., 2012). Those who were diagnosed with AD needed greater temperatures to elicit pain when compared to cognitively normal participants. Even if pain proves to be a useful early marker, the difference found in this dissertation suggests further research is warranted into how AD pathology disrupts the pain experience. Chronic pain is one of the leading comorbidities with AD and difficult to treat in patients with $\mathrm{AD}$. Others have shown $\mathrm{AD}$ patients get/receive fewer pain medicines compared to healthy controls (T. B. Monroe, Carter, et al., 2013). The fact that there are limited options available for chronic pain management in older adults with AD provides direction from this dissertation that may lead to novel drug therapies. 


\section{List of References}

"2019 Alzheimer's disease facts and figures" (2019). In: Alzheimer's \& Dementia 15.3, pp. 321-387. ISSN: 15525260. DOI: 10 .1016/ j . jalz . 2019 . 01 .010. URL: http: //www . sciencedirect.com/science/article/pii/S1552526019300317.

"2020 Alzheimer's disease facts and figures" (2020). In: Alzheimers Dement 16.3, pp. 391460. ISSN: 1552-5279 (Electronic) 1552-5260 (Linking). DOI: 10 . 1002 / alz . 12068. URL: https://www.ncbi.nlm.nih.gov/pubmed/32157811.

Abdulla, A. et al. (2013). "Evidence-based clinical practice guidelines on management of pain in older people". In: Age Ageing 42.2, pp. 151-3. ISSN: 1468-2834 (Electronic) 00020729 (Linking). DOI: 10 . 1093/ageing/afs199. URL: https : / www . ncbi .nlm.nih.gov/ pubmed/23335785.

Andersen, R. M. (2008). "National health surveys and the behavioral model of health services use". In: Med Care 46.7, pp. 647-53. ISSN: 0025-7079 (Print) 0025-7079 (Linking). DOI: 10 .1097/MLR.0b013e31817a835d. URL: https ://www .ncbi.nlm.nih.gov/pubmed/ 18580382.

Association, Alzheimer's (2018). "Facts and Figures". In: URL: https : / / www . alzheimersanddementia.com/article/S1552-5260(18)30041-4/fulltext\#sec7.3.

Balachandar, R. et al. (2017). "Resting-State Functional Connectivity Changes Associated with Visuospatial Cognitive Deficits in Patients with Mild Alzheimer Disease". In: Dement Geriatr Cogn Disord 43.5-6, pp. 229-236. ISSN: 1421-9824 (Electronic) 1420-8008 (Linking). DOI: 10 .1159/000457118. URL: https : / /ww . ncbi .nlm . nih . gov/pubmed/ 28351035.

Barker, W. W. et al. (2002). "Relative frequencies of Alzheimer disease, Lewy body, vascular and frontotemporal dementia, and hippocampal sclerosis in the State of Florida Brain Bank". In: Alzheimer Dis Assoc Disord 16.4, pp. 203-12. ISSN: 0893-0341 (Print) 0893-0341 (Linking). DOI: 10 . 1097 / 00002093 - 200210000-00001. URL: https : / / www . ncbi . nlm . nih.gov/pubmed/12468894.

Benedetti, F. et al. (1999). "Pain threshold and tolerance in Alzheimer's disease". In: Pain 80.1-2, pp. 377-82. ISSN: 0304-3959 (Print) 0304-3959 (Linking). DOI: 10 . 1016 / s0304 3959 (98)00228-0. URL: https://www.ncbi.nlm.nih.gov/pubmed/10204751.

Boise, L. (2006). "Improving dementia through physician education: some challenges". In: Clinical Gerontologist 29.2, 3-10 8p. ISSN: 0731-7115. URL: http : / search . ebscohost . $\mathrm{com} / \log$ in. aspx?direct=true\&db=cin20\&AN=106228358\&site=ehost - live. 
Boise, L. et al. (1999). "Diagnosing dementia: perspectives of primary care physicians". In: Gerontologist 39.4, pp. 457-64. ISSN: 0016-9013 (Print) 0016-9013 (Linking). DOI: 10 . 1093/ geront/39.4.457. URL: https://www.ncbi.nlm.nih.gov/pubmed/10495584.

Boustani, M. et al. (2005). "Implementing a screening and diagnosis program for dementia in primary care". In: J Gen Intern Med 20.7, pp. 572-7. ISSN: 1525-1497 (Electronic) 08848734 (Linking). DOI: 10 .1111/j . 1525-1497 . 2005 .0126 . x. URL: https : / / www . ncbi . nlm.nih.gov/pubmed/16050849.

Boyle, P. A. et al. (2007). "Lower extremity motor function and disability in mild cognitive impairment". In: Exp Aging Res 33.3, pp. 355-71. ISSN: 0361-073X (Print) 0361-073X (Linking). DOI: 10 . 1080/03610730701319210. URL: https : / www . ncbi . nlm.nih . gov/ pubmed/17497375.

Braak, H. et al. (2003). "Staging of brain pathology related to sporadic Parkinson's disease". In: Neurobiol Aging 24.2, pp. 197-211. ISSN: 0197-4580 (Print) 0197-4580 (Linking). DOI: 10.1016/s0197-4580 (02) 00065-9. URL: https : / /www . ncbi .nlm . nih . gov/pubmed / 12498954.

Bradford, A. et al. (2009). "Missed and delayed diagnosis of dementia in primary care: prevalence and contributing factors". In: Alzheimer Dis Assoc Disord 23.4, pp. 306-14. ISSN: 1546-4156 (Electronic) 0893-0341 (Linking). DOI: 10 . 1097/WAD . 0b013e3181a6bebc. URL: https://www.ncbi.nlm.nih.gov/pubmed/19568149.

Camicioli, R. et al. (1998). "Motor slowing precedes cognitive impairment in the oldest old". In: Neurology 50.5, pp. 1496-8. ISSN: 0028-3878 (Print) 0028-3878 (Linking). DOI: 10.1212/wnl.50.5.1496. URL: https://www.ncbi.nlm.nih.gov/pubmed/9596020.

Chou, H. K. et al. (2012). "A pilot study of the telecare medical support system as an intervention in dementia care: the views and experiences of primary caregivers". In: J Nurs Res 20.3, pp. 169-80. ISSN: 1948-965X (Electronic) 1682-3141 (Linking). DOI: 10 . 1097 / jnr.0b013e318263d916. URL: https://www.ncbi.nlm.nih.gov/pubmed/22902976.

Cleeland, C. S. and K. M. Ryan (1994). "Pain assessment: global use of the Brief Pain Inventory". In: Ann Acad Med Singap 23.2, pp. 129-38. ISSN: 0304-4602 (Print) 0304-4602 (Linking). URL: https://www.ncbi.nlm.nih.gov/pubmed/8080219.

Cole, L. J. et al. (2006). "Pain sensitivity and fMRI pain-related brain activity in Alzheimer's disease". In: Brain 129.Pt 11, pp. 2957-65. ISSN: 1460-2156 (Electronic) 0006-8950 (Linking). DOI: 10 . 1093/brain/aw1228. URL: https : / / www . ncbi .nlm . nih . gov/pubmed / 16951408.

Corbett, A. et al. (2014). "The importance of pain management in older people with dementia". In: Br Med Bull 111.1, pp. 139-48. ISSN: 1471-8391 (Electronic) 0007-1420 (Linking). DOI: $10.1093 / \mathrm{bmb} / \mathrm{ldu} 023$. URL: https://www.ncbi.nlm.nih.gov/pubmed/25190763.

Cowan, R. L. et al. (2017). "Sex Differences in the Psychophysical Response to Contact Heat in Moderate Cognitive Impairment Alzheimer's Disease: A Cross-Sectional Brief Report". In: J Alzheimers Dis 60.4, pp. 1633-1640. ISSN: 1875-8908 (Electronic) 1387-2877 (Linking). DOI: 10.3233/JAD-170532. URL: https : / /www .ncbi .nlm.nih.gov/pubmed/ 28968238. 
Cummings, J. et al. (2019). “Alzheimer's disease drug development pipeline: 2019". In: Alzheimers Dement (N Y) 5, pp. 272-293. ISSN: 2352-8737 (Electronic) 2352-8737 (Linking). DOI: 10.1016/j .trci.2019.05.008. URL: https : / www . ncbi .nlm.nih . gov/pubmed/ 31334330.

Dhilla Albers, A. et al. (2016). "Episodic memory of odors stratifies Alzheimer biomarkers in normal elderly". In: Ann Neurol 80.6, pp. 846-857. ISSN: 1531-8249 (Electronic) 03645134 (Linking). DOI: 10 . 1002 / ana . 24792. URL: https : / / www . ncbi . nlm . nih . gov / pubmed/27696605.

Dubois, B., H. H. Feldman, et al. (2007). "Research criteria for the diagnosis of Alzheimer's disease: revising the NINCDS-ADRDA criteria". In: Lancet Neurol 6.8, pp. 734-46. ISSN: 1474-4422 (Print) 1474-4422 (Linking). DOI: 10 . 1016 / S1474 - 4422 (07 ) 70178 - 3. URL: https://www.ncbi.nlm.nih.gov/pubmed/17616482.

Dubois, B., A. Padovani, et al. (2016). “Timely Diagnosis for Alzheimer's Disease: A Literature Review on Benefits and Challenges". In: J Alzheimers Dis 49.3, pp. 617-31. ISSN: 1875-8908 (Electronic) 1387-2877 (Linking). DOI: 10 . 3233 / JAD - 150692. URL: https : //www.ncbi.nlm.nih.gov/pubmed/26484931.

Edwards, R. R., R. B. Fillingim, and T. J. Ness (2003). “Age-related differences in endogenous pain modulation: a comparison of diffuse noxious inhibitory controls in healthy older and younger adults". In: Pain 101.1-2, pp. 155-65. ISSN: 0304-3959 (Print) 03043959 (Linking). DOI: 10 .1016/s0304-3959 (02) 00324-x. URL: https : //www . ncbi .nlm . nih.gov/pubmed/12507710.

Edwards, R., S. E. Voss, and S. Iliffe (2015). "The development and evaluation of an educational intervention for primary care promoting person-centred responses to dementia". In: Dementia (London) 14.4, pp. 468-82. ISSN: 1741-2684 (Electronic) 1471-3012 (Linking). DOI: 10 . 1177 / 1471301213499768. URL: https : / / www . ncbi . nlm . nih . gov / pubmed / 24339110.

Eichler, T. et al. (2014). "The benefits of implementing a computerized interventionmanage-ment-system (IMS) on delivering integrated dementia care in the primary care setting". In: Int Psychogeriatr 26.8, pp. 1377-85. ISSN: 1741-203X (Electronic) 1041-6102 (Linking). DOI: 10.1017/S1041610214000830. URL: https : / www . ncbi.nlm.nih.gov/ pubmed/24811145.

Esiri, M. M. and G. K. Wilcock (1984). “The olfactory bulbs in Alzheimer's disease”. In: J Neurol Neurosurg Psychiatry 47.1, pp. 56-60. ISSN: 0022-3050 (Print) 0022-3050 (Linking). DOI: 10.1136/jnnp.47.1.56. URL: https://www.ncbi.nlm.nih.gov/pubmed/6693914.

Fillingim, R. B. et al. (2009). "Sex, gender, and pain: a review of recent clinical and experimental findings". In: J Pain 10.5, pp. 447-85. ISSN: 1528-8447 (Electronic) 1526-5900 (Linking). DOI: 10.1016/j . jpain.2008.12.001. URL: https://www.ncbi.nlm.nih.gov/ pubmed/19411059.

Folstein, M. F., S. E. Folstein, and P. R. McHugh (1975). "'Mini-mental state". A practical method for grading the cognitive state of patients for the clinician". In: J Psychiatr 
Res 12.3, pp. 189-98. ISSN: 0022-3956 (Print) 0022-3956 (Linking). DOI: 10 . 1016/0022 3956 (75) $90026-6$.

Fortinsky, R. H. et al. (2010H). “Primary care physicians' dementia care practices: evidence of geographic variation". In: Gerontologist 50.2, pp. 179-91. ISSN: 1758-5341 (Electronic) 0016-9013 (Linking). DOI: 10 . 1093/geront/gnp106. URL: https : / www . ncbi .nlm .nih . gov/pubmed/19597058.

Gibson, S. J. et al. (2001). “An examination of pain perception and cerebral event-related potentials following carbon dioxide laser stimulation in patients with Alzheimer's disease and age-matched control volunteers". In: Pain Res Manag 6.3, pp. 126-32. ISSN: 12036765 (Print) 1203-6765 (Linking). DOI: 10 .1155/2001/814374. URL: https : //www . ncbi . nlm.nih.gov/pubmed/11854775.

Growdon, M. E. et al. (2015). “Odor identification and Alzheimer disease biomarkers in clinically normal elderly". In: Neurology 84.21, pp. 2153-60. ISSN: 1526-632X (Electronic) 0028-3878 (Linking). DOI: 10 . 1212/WNL . 0000000000001614 . URL: https : / /ww . ncbi . nlm.nih.gov/pubmed/25934852.

Grypma, L. (2007). “Decision support for primary care clinicians improves process of care but not symptoms in people with depression". In: Evidence Based Mental Health 10.2, 4747 1p. ISSN: 1362-0347. URL: http: //search. ebscohost. com/login. aspx?direct=true\& $\mathrm{db}=\mathrm{cin} 20 \& \mathrm{AN}=106198957 \&$ site $=$ ehost - live.

Haasum, Y., J. Fastbom, L. Fratiglioni, and K. Johnell (2012). "Undertreatment of osteoporosis in persons with dementia? A population-based study". In: Osteoporos Int 23.3, pp. 1061-8. ISSN: 1433-2965 (Electronic) 0937-941X (Linking). DOI: 10 . 1007 / s00198 011-1636-8. URL: https://www.ncbi.nlm.nih.gov/pubmed/21499775.

Haasum, Y., J. Fastbom, L. Fratiglioni, I. Kareholt, et al. (2011). "Pain treatment in elderly persons with and without dementia: a population-based study of institutionalized and home-dwelling elderly". In: Drugs Aging 28.4, pp. 283-93. ISSN: 1179-1969 (Electronic) 1170-229X (Linking). DOI: 10 . 2165/11587040 - 000000000-00000. URL: https : / /ww . ncbi.nlm.nih.gov/pubmed/21428463.

Hamilton, M. (1960). “A rating scale for depression”. In: J Neurol Neurosurg Psychiatry 23, pp. 56-62. ISSN: 0022-3050 (Print) 0022-3050 (Linking). DOI: 10 . $1136 /$ jnnp . 23 . 1 . 56. URL: https://www.ncbi.nlm.nih.gov/pubmed/14399272.

Hebert, L. E. et al. (2013). "Alzheimer disease in the United States (2010-2050) estimated using the 2010 census". In: Neurology 80.19, pp. 1778-83. ISSN: 1526-632X (Electronic) 0028-3878 (Linking). DOI: 10 . 1212/WNL . Ob013e31828726f5. URL: https : / /ww . ncbi . nlm.nih.gov/pubmed/23390181.

Hippius, H. and G. Neundorfer (2003). "The discovery of Alzheimer's disease". In: Dialogues Clin Neurosci 5.1, pp. 101-8. ISSN: 1294-8322 (Print) 1294-8322 (Linking). URL: https://www.ncbi.nlm.nih.gov/pubmed/22034141.

Hollingshead, August B (1975). "Four factor index of social status". In: 
Hunt, S.P. and M. Koltzenburg (2005). The Neurobiology of Pain: (molecular and Cellular Neurobiology). Oxford University Press. ISBN: 9780198515616. URL: https ://books . google. com/books?id=plJ8rZCQ85MC.

Iliffe, S., L. Robinson, et al. (2009). "Primary care and dementia: 1. diagnosis, screening and disclosure". In: International Journal of Geriatric Psychiatry 24.9, pp. 895-901. ISSN: 0885-6230. DOI: 10 . 1002 / gps . 2204. URL: http : / / proxy . library . vanderbilt . edu / login? url=http: // search . ebscohost. com/login . aspx ? direct=true \& db=ccm\&AN= 105417702\&site=ehost - live\&scope=site.

Iliffe, S., J. Wilcock, et al. (2010). "Evidence-based interventions in dementia: A pragmatic cluster-randomised trial of an educational intervention to promote earlier recognition and response to dementia in primary care (EVIDEM-ED)". In: Trials 11, p. 13. ISSN: 17456215 (Electronic) 1745-6215 (Linking). DOI: 10 . 1186/1745-6215-11-13. URL: https : //www.ncbi.nlm.nih.gov/pubmed/20146803.

Jack C. R., Jr. et al. (2018). “NIA-AA Research Framework: Toward a biological definition of Alzheimer's disease". In: Alzheimers Dement 14.4, pp. 535-562. ISSN: 1552-5279 (Electronic) 1552-5260 (Linking). DOI: 10 . $1016 / \mathrm{j}$. jalz . 2018 . 02 . 018. URL: https : //www.ncbi.nlm.nih.gov/pubmed/29653606.

Jeste, D. V. et al. (2007). "A new brief instrument for assessing decisional capacity for clinical research". In: Arch Gen Psychiatry 64.8, pp. 966-74. ISSN: 0003-990X (Print) 0003-990X (Linking). DOI: 10.1001/archpsyc.64.8.966. URL: https : / www .ncbi.nlm.nih.gov/ pubmed/17679641.

Jimbo, D. et al. (2011). "Specific feature of olfactory dysfunction with Alzheimer's disease inspected by the Odor Stick Identification Test". In: Psychogeriatrics 11.4, pp. 196-204. ISSN: 1479-8301 (Electronic) 1346-3500 (Linking). DOI: 10 . 1111/ j . 1479-8301 . 2011 . 00387 .x. URL: https://www.ncbi.nlm.nih.gov/pubmed/22151238.

Johannes, C. B. et al. (2010). "The prevalence of chronic pain in United States adults: results of an Internet-based survey". In: J Pain 11.11, pp. 1230-9. ISSN: 1528-8447 (Electronic) 1526-5900 (Linking). DOI: 10 . 1016/ j . jpain . 2010 . 07 . 002. URL: https : / / www . ncbi . nlm.nih.gov/pubmed/20797916.

Jones, A. K., B. Kulkarni, and S. W. Derbyshire (2003). "Pain mechanisms and their disorders". In: Br Med Bull 65, pp. 83-93. ISSN: 0007-1420 (Print) 0007-1420 (Linking). DOI: 10.1093/bmb/65.1.83. URL: https://www.ncbi.nlm.nih.gov/pubmed/12697618.

Knopman, D., J. A. Donohue, and E. M. Gutterman (2000). "Patterns of care in the early stages of Alzheimer's disease: impediments to timely diagnosis". In: J Am Geriatr Soc 48.3, pp. 300-4. ISSN: 0002-8614 (Print) 0002-8614 (Linking). DOI: 10 . 1111 / j . 1532 5415.2000.tb02650.x. URL: https://www.ncbi.nlm.nih.gov/pubmed/10733057.

Koch, T. and S. Iliffe (2011). "Dementia diagnosis andmanagement: A narrative review of changing practice". In: British Journal of General Practice 61.589, e513-e525. URL: http : //ovidsp. ovid.com/ovidweb. cgi? T=JS\&CSC=Y\&NEWS=N\&PAGE=fulltext\&D=emed10\&AN= 2011682974. 
Kooten, J. van et al. (2015). "Pain in dementia: prevalence and associated factors: protocol of a multidisciplinary study". In: BMC Geriatr 15, p. 29. ISSN: 1471-2318 (Electronic) 14712318 (Linking). DOI: 10.1186/s12877-015-0025-0. URL: https : //www .ncbi .nlm.nih . gov/pubmed/25879681.

Kotagal, V. et al. (2015). "Factors associated with cognitive evaluations in the United States". In: Neurology 84.1, pp. 64-71. ISSN: 1526-632X (Electronic) 0028-3878 (Linking). DOI: 10.1212/WNL . 0000000000001096 . URL: https ://wWw .ncbi.nlm.nih.gov/pubmed/ 25428689.

Kouzuki, M. et al. (2018). "Comparison of olfactory and gustatory disorders in Alzheimer's disease". In: Neurol Sci 39.2, pp. 321-328. ISSN: 1590-3478 (Electronic) 1590-1874 (Linking). DOI: 10 . 1007 / s10072-017-3187-z. URL: https : / / www . ncbi .nlm . nih . gov / pubmed/29128987.

Kromer Vogt, L. J. et al. (1990). “Pathological alterations in the amygdala in Alzheimer's disease". In: Neuroscience 37.2, pp. 377-85. ISSN: 0306-4522 (Print) 0306-4522 (Linking). DOI: $10.1016 / 0306-4522(90) 90408-v$.

Kumar, A., A. Singh, and Ekavali (2015). "A review on Alzheimer's disease pathophysiology and its management: an update". In: Pharmacol Rep 67.2, pp. 195-203. ISSN: 17341140 (Print) 1734-1140 (Linking). DOI: 10 . 1016/ j . pharep. 2014 . 09 .004. URL: https : //www.ncbi.nlm.nih.gov/pubmed/25712639.

Kunz, M., V. Mylius, et al. (2009). "Influence of dementia on multiple components of pain". In: Eur J Pain 13.3, pp. 317-25. ISSN: 1532-2149 (Electronic) 1090-3801 (Linking). DOI: 10. 1016/j.ejpain. 2008.05.001. URL: https://www.ncbi.nlm.nih.gov/pubmed/18562225.

Kunz, M., S. Scharmann, et al. (2007). "The facial expression of pain in patients with dementia". In: Pain 133.1-3, pp. 221-8. ISSN: 1872-6623 (Electronic) 0304-3959 (Linking). DOI: 10 . 1016/j . pain . 2007 . 09 .007. URL: https : / www . ncbi .nlm.nih.gov/pubmed/ 17949906.

Kurlowicz, L. (1999). “The Geriatric Depression Scale (GDS)”. In: Geriatr Nurs 20.4, pp. 2123. ISSN: 0197-4572 (Print) 0197-4572 (Linking). URL: https : / www . ncbi . nlm . nih . gov/ pubmed/10711094.

Lafaille-Magnan, M. E. et al. (2017). “Odor identification as a biomarker of preclinical AD in older adults at risk". In: Neurology 89.4, pp. 327-335. ISSN: 1526-632X (Electronic) 00283878 (Linking). DOI: 10 . 1212/WNL . 0000000000004159 . URL: https : / www . ncbi . nlm . nih.gov/pubmed/28659431.

Lang, C. J. et al. (2006). "Taste in dementing diseases and parkinsonism". In: J Neurol Sci 248.1-2, pp. 177-84. ISSN: 0022-510X (Print) 0022-510X (Linking). DOI: $10.1016 / \mathrm{j}$. jns . 2006.05.020. URL: https://www.ncbi.nlm.nih.gov/pubmed/16769086.

Laptinskaya, D. et al. (2018). “Auditory Memory Decay as Reflected by a New Mismatch Negativity Score Is Associated with Episodic Memory in Older Adults at Risk of Dementia". In: Front Aging Neurosci 10, p. 5. ISSN: 1663-4365 (Print) 1663-4365 (Linking). DOI: 10.3389/fnagi.2018.00005. URL: https://www.ncbi.nlm.nih.gov/pubmed/29456500. 
Leveille, S. G. et al. (2005). "Sex differences in musculoskeletal pain in older adults". In: Pain 116.3, pp. 332-8. ISSN: 0304-3959 (Print) 0304-3959 (Linking). DOI: 10 . 1016/j . pain. 2005.05.002. URL: https://www.ncbi.nlm.nih.gov/pubmed/15982814.

Long, J. M. and D. M. Holtzman (2019). "Alzheimer Disease: An Update on Pathobiology and Treatment Strategies". In: Cell 179.2, pp. 312-339. ISSN: 1097-4172 (Electronic) 00928674 (Linking). DOI: 10.1016/j . cell . 2019.09.001. URL: https : //www . ncbi.nlm.nih. gov/pubmed/31564456.

Maehlen, M. T. et al. (2013). "Associations between APOE genotypes and disease susceptibility, joint damage and lipid levels in patients with rheumatoid arthritis". In: PLoS One 8.4, e60970. ISSN: 1932-6203 (Electronic) 1932-6203 (Linking).

Magin, P. et al. (2016). "Attitudes to Alzheimer's disease testing of Australian general practice patients: a cross-sectional questionnaire-based study". In: Int J Geriatr Psychiatry 31.4, pp. 361-6. ISSN: 1099-1166 (Electronic) 0885-6230 (Linking). DOI: 10 . 1002 / gps . 4335. URL: https://www.ncbi.nlm.nih.gov/pubmed/26258761.

Mahley, R. W. and Jr. Rall S. C. (2000). "Apolipoprotein E: far more than a lipid transport protein". In: Annu Rev Genomics Hum Genet 1, pp. 507-37. ISSN: 1527-8204 (Print) 15278204 (Linking). DOI: 10.1146/annurev.genom.1.1.507.

Martin, S., J. Fleming, et al. (2015). “Exploring attitudes and preferences for dementia screening in Britain: contributions from carers and the general public". In: BMC Geriatr 15.1, p. 110. ISSN: 1471-2318 (Electronic) 1471-2318 (Linking). DOI: 10.1186/s12877015-0100-6. URL: https://www.ncbi.nlm.nih.gov/pubmed/26354754.

Martin, S., S. Kelly, et al. (2015). "Attitudes and preferences towards screening for dementia: a systematic review of the literature". In: BMC Geriatr 15, p. 66. ISSN: 1471-2318 (Electronic) 1471-2318 (Linking). DOI: 10 . 1186/s12877-015-0064-6. URL: https : / / www . ncbi.nlm.nih.gov/pubmed/26076729.

Masaoka, Y. et al. (2013). "Markers of brain illness may be hidden in your olfactory ability: a Japanese perspective". In: Neurosci Lett 549, pp. 182-5. ISSN: $1872-7972$ (Electronic) 0304-3940 (Linking). DOI: 10 . 1016/ j . neulet . 2013 . 05 .077. URL: https : / www . ncbi . nlm.nih.gov/pubmed/23769725.

Matlin, Margaret W. and Hugh J. Foley (1992). Sensation and perception, 3rd ed. Sensation and perception, 3rd ed. Needham Heights, MA, US: Allyn \& Bacon, pp. xix, 533-xix, 533. ISBN: 0-205-13313-4 (Hardcover).

Mok, W. et al. (2004). "Clinicopathological concordance of dementia diagnoses by community versus tertiary care clinicians". In: Am J Alzheimers Dis Other Demen 19.3, pp. 161-5. ISSN: 1533-3175 (Print) 1533-3175 (Linking). DOI: 10.1177 / 153331750401900309. URL: https://www.ncbi.nlm.nih.gov/pubmed/15214202.

Monroe, T. B., P. A. Beach, et al. (2017). “The Impact of Alzheimer's Disease on the Resting State Functional Connectivity of Brain Regions Modulating Pain: A Cross Sectional Study". In: J Alzheimers Dis 57.1, pp. 71-83. ISSN: 1875-8908 (Electronic) 1387-2877 (Linking). DOI: 10 . 3233 / JAD - 161187. URL: https : / / www . ncbi . nlm . nih . gov / pubmed / 28222526. 
Monroe, T. B., M. A. Carter, et al. (2013). "Pain and hospice care in nursing home residents with dementia and terminal cancer". In: Geriatr Gerontol Int 13.4, pp. 1018-25. ISSN: 14470594 (Electronic) 1447-0594 (Linking). DOI: 10 . 1111/ggi . 12049. URL: https : / / www . ncbi.nlm.nih.gov/pubmed/23506621.

Monroe, T. B., R. B. Fillingim, et al. (2018). "Sex Differences in Brain Regions Modulating Pain Among Older Adults: A Cross-Sectional Resting State Functional Connectivity Study". In: Pain Med 19.9, pp. 1737-1747. ISSN: 1526-4637 (Electronic) 1526-2375 (Linking). DOI: 10 . 1093 / pm / pnx084. URL: https : / / www . ncbi . nlm . nih . gov / pubmed / 28505337.

Monroe, T. B., S. J. Gibson, et al. (2016). "Contact heat sensitivity and reports of unpleasantness in communicative people with mild to moderate cognitive impairment in Alzheimer's disease: a cross-sectional study". In: BMC Med 14, p. 74. ISSN: 1741-7015 (Electronic) 1741-7015 (Linking). DOI: 10 . 1186 / s12916-016-0619-1. URL: https : //www.ncbi.nlm.nih.gov/pubmed/27164846.

Monroe, T. B., J. C. Gore, S. P. Bruehl, et al. (2015). "Sex differences in psychophysical and neurophysiological responses to pain in older adults: a cross-sectional study". In: Biol Sex Differ 6, p. 25. ISSN: 2042-6410 (Print) 2042-6410 (Linking). DOI: 10 . 1186 /s13293 015-0041-y. URL: https://www.ncbi.nlm.nih.gov/pubmed/26579217.

Monroe, T. B., J. C. Gore, L. M. Chen, et al. (2012). “Pain in people with Alzheimer disease: potential applications for psychophysical and neurophysiological research". In: J Geriatr Psychiatry Neurol 25.4, pp. 240-55. ISSN: 0891-9887 (Print) 0891-9887 (Linking). DOI: 10. 1177/0891988712466457. URL: https://www.ncbi.nlm.nih.gov/pubmed/23277361.

Monroe, T. B., K. A. Herr, et al. (2013). "Ethical and legal issues in pain research in cognitively impaired older adults". In: Int J Nurs Stud 50.9, pp. 1283-7. ISSN: 1873-491X (Electronic) 0020-7489 (Linking). DOI: 10 .1016/j . i jnurstu .2012 .11 .023. URL: https : //www.ncbi.nlm.nih.gov/pubmed/23245707.

Morrison, R. S. et al. (2003). "The impact of post-operative pain on outcomes following hip fracture". In: Pain 103.3, pp. 303-11. ISSN: 0304-3959 (Print) 0304-3959 (Linking). DOI: 10 . 1016/s0304-3959 (02) 00458-x. URL: https : / / www . ncbi .nlm . nih . gov/pubmed/ 12791436.

Mortamais, M. et al. (2017). “Detecting cognitive changes in preclinical Alzheimer's disease: A review of its feasibility". In: Alzheimers Dement 13.4, pp. 468-492. ISSN: 1552-5279 (Electronic) 1552-5260 (Linking). DOI: 10 . $1016 / \mathrm{j}$. jalz . 2016 . 06 . 2365. URL: https : //www.ncbi.nlm.nih.gov/pubmed/27702618.

Moyer, V. A. and U. S. Preventive Services Task Force (2014). “Screening for cognitive impairment in older adults: U.S. Preventive Services Task Force recommendation statement". In: Ann Intern Med 160.11, pp. 791-7. ISSN: 1539-3704 (Electronic) 0003-4819 (Linking). DOI: 10.7326/M14-0496. URL: https://www.ncbi.nlm.nih.gov/pubmed/24663815.

Murphy, C. (2019). "Olfactory and other sensory impairments in Alzheimer disease". In: Nat Rev Neurol 15.1, pp. 11-24. ISSN: 1759-4766 (Electronic) 1759-4758 (Linking). DOI: 10. 1038/s41582-018-0097-5. URL: https://www.ncbi.nlm.nih.gov/pubmed/30532084. 
Najm, R., E. A. Jones, and Y. Huang (2019). “Apolipoprotein E4, inhibitory network dysfunction, and Alzheimer's disease". In: Mol Neurodegener 14.1, p. 24. ISSN: 1750-1326 (Electronic) 1750-1326 (Linking). DOI: 10 . $1186 /$ s13024-019-0324-6. URL: https : //www.ncbi.nlm.nih.gov/pubmed/31186040.

Naudin, M. et al. (2015). “Taste identification used as a potential discriminative test among depression and Alzheimers disease in elderly: A pilot study". In: Psychiatry Res 228.2, pp. 228-32. ISSN: 1872-7123 (Electronic) 0165-1781 (Linking). DOI: 10.1016/j . psychres . 2015.03.021. URL: https://www.ncbi.nlm.nih.gov/pubmed/25998001.

Naugle, K. M. et al. (2013). "Offset analgesia is reduced in older adults". In: Pain 154.11, pp. 2381-7. ISSN: 1872-6623 (Electronic) 0304-3959 (Linking). DOI: 10 . $1016 / \mathrm{j} \cdot$ pain . 2013.07.015. URL: https://www.ncbi.nlm.nih.gov/pubmed/23872117.

Olofsson, J. et al. (2010). “Odor identification impairment in carriers of ApoE e4 is independent of clinical dementia". In: 31.4, pp. 567-577. ISSN: 0197-4580. DOI: 10 .10162008.05. 01. URL: http://www. sciencedirect.com/science/article/pii/S0197458008001723.

Parvizi, J., G. W. Van Hoesen, and A. Damasio (2000). "Selective pathological changes of the periaqueductal gray matter in Alzheimer's disease". In: Ann Neurol 48.3, pp. 344-53. ISSN: 0364-5134 (Print) 0364-5134 (Linking). URL: https : / / www . ncbi . nlm . nih . gov / pubmed/10976641.

Patterson, C (2018). “World Alzheimer Report 2018: The State of the Art of Dementia Research: New Frontiers". In: Alzheimer's Disease International (ADI): London, UK.

Paulson, C. M., T. Monroe, and L. C. Mion (2014). "Pain assessment in hospitalized older adults with dementia and delirium". In: J Gerontol Nurs 40.6, pp. 10-5. ISSN: 0098-9134 (Print) 0098-9134 (Linking). DOI: 10 . 3928/00989134-20140428-02. URL: https : / www . ncbi.nlm.nih.gov/pubmed/24800815.

Paulson, P. E. et al. (1998). "Gender differences in pain perception and patterns of cerebral activation during noxious heat stimulation in humans". In: Pain 76.1-2, pp. 223-9. ISSN: 0304-3959 (Print) 0304-3959 (Linking). DOI: 10 . 1016 / s0304 - 3959 (98 ) 00048 - 7. URL: https://www.ncbi.nlm.nih.gov/pubmed/9696477.

Perry, M. et al. (2008). "An in-home geriatric programme for vulnerable communitydwelling older people improves the detection of dementia in primary care". In: Int J Geriatr Psychiatry 23.12, pp. 1312-9. ISSN: 1099-1166 (Electronic) 0885-6230 (Linking). DOI: $10.1002 /$ gps . 2128. URL: https://www .ncbi.nlm.nih.gov/pubmed/18853470.

Peters, J. M. et al. (2003). “Olfactory function in mild cognitive impairment and Alzheimer's disease: an investigation using psychophysical and electrophysiological techniques". In: Am J Psychiatry 160.11, pp. 1995-2002. ISSN: 0002-953X (Print) 0002-953X (Linking). DOI: 10.1176/appi .ajp.160.11.1995. URL: https : //www .ncbi .nlm.nih . gov/pubmed/14594747.

Petzke, F. et al. (2005). "Differences in unpleasantness induced by experimental pressure pain between patients with fibromyalgia and healthy controls". In: Eur J Pain 9.3, pp. 325-35. ISSN: 1090-3801 (Print) 1090-3801 (Linking). DOI: 10 . 1016/ j . ejpain . 2004 . 09.001. 
Quarmley, M. et al. (2017). “Odor Identification Screening Improves Diagnostic Classification in Incipient Alzheimer's Disease". In: J Alzheimers Dis 55.4, pp. 1497-1507. ISSN: 1875-8908 (Electronic) 1387-2877 (Linking). DOI: 10 . 3233 / JAD - 160842. URL: https : //www.ncbi.nlm.nih.gov/pubmed/27886011.

Riley J. L., 3rd et al. (2010). "Lack of endogenous modulation and reduced decay of prolonged heat pain in older adults". In: Pain 150.1, pp. 153-60. ISSN: 1872-6623 (Electronic) 0304-3959 (Linking). DOI: 10 .1016/j . pain. 2010.04 .020. URL: https : //www .ncbi .nlm. nih.gov/pubmed/20546997.

Risacher, S. L. and A. J. Saykin (2013). "Neuroimaging biomarkers of neurodegenerative diseases and dementia". In: Semin Neurol 33.4, pp. 386-416. ISSN: 1098-9021 (Electronic) 0271-8235 (Linking). DOI: 10.1055/s-0033-1359312. URL: https : //www.ncbi.nlm.nih. gov/pubmed/24234359.

Risacher, S. L., D. Wudunn, et al. (2013). "Visual contrast sensitivity in Alzheimer's disease, mild cognitive impairment, and older adults with cognitive complaints". In: Neurobiol Aging 34.4, pp. 1133-44. ISSN: 1558-1497 (Electronic) 0197-4580 (Linking). DOI: 10.1016/ j . neurobiolaging . 2012 .08 .007. URL: https : / / www . ncbi.nlm . nih . gov/pubmed / 23084085.

Robinson, L., E. Tang, and J. P. Taylor (2015). “Dementia: timely diagnosis and early intervention". In: BMJ 350, h3029. ISSN: 1756-1833 (Electronic) 0959-8138 (Linking). DOI: 10.1136/bmj . h3029. URL: https://www.ncbi.nlm.nih.gov/pubmed/26079686.

Romano R. R., 3rd, M. A. Carter, A. R. Anderson, et al. (2020). "An integrative review of system-level factors influencing dementia detection in primary care". In: J Am Assoc Nurse Pract 32.4, pp. 299-305. ISSN: 2327-6924 (Electronic) 2327-6886 (Linking). DOI: 10 . 1097/JXX.0000000000000230. URL: https://www.ncbi.nlm.nih.gov/pubmed/31274678.

Romano R. R., 3rd, M. A. Carter, and T. B. Monroe (2020). "Narrative Review of Sensory Changes as a Biomarker for Alzheimer's Disease". In: Biol Res Nurs, p. 1099800420947176. ISSN: 1552-4175 (Electronic) 1099-8004 (Linking). DOI: 10 . 1177 / 1099800420947176. URL: https://www.ncbi.nlm.nih.gov/pubmed/32799655.

Romano, R. R. et al. (2019). "Sex Differences in Associations of Cognitive Function with Perceptions of Pain in Older Adults". In: J Alzheimers Dis 70.3, pp. 715-722. ISSN: 18758908 (Electronic) 1387-2877 (Linking). DOI: 10 . 3233 / JAD - 190142. URL: https : / www . ncbi.nlm.nih.gov/pubmed/31256133.

Safieh, M., A. D. Korczyn, and D. M. Michaelson (2019). "ApoE4: an emerging therapeutic target for Alzheimer's disease". In: BMC Med 17.1, p. 64. ISSN: 1741-7015 (Electronic) 1741-7015 (Linking). DOI: 10.1186/s12916-019-1299-4. URL: https : / www .ncbi .nlm. nih.gov/pubmed/30890171.

Scherder, E. J., J. A. Sergeant, and D. F. Swaab (2003). “Pain processing in dementia and its relation to neuropathology". In: Lancet Neurol 2.11, pp. 677-86. ISSN: 1474-4422 (Print) 1474-4422 (Linking). DOI: 10 .1016/s1474-4422(03) 00556-8. URL: https : / www . ncbi . nlm.nih.gov/pubmed/14572736. 
Selden, N. et al. (1994). "Human striatum: chemoarchitecture of the caudate nucleus, putamen and ventral striatum in health and Alzheimer's disease". In: Neuroscience 60.3, pp. 621-36. ISSN: 0306-4522 (Print) 0306-4522 (Linking). DOI: 10 . 1016/0306 - 4522 (94) 90491-x. URL: https://www.ncbi.nlm.nih.gov/pubmed/7523983.

Serby, M. et al. (1996). “Olfactory identification deficits in relatives of Alzheimer's disease patients". In: Biol Psychiatry 39.5, pp. 375-7. ISSN: 0006-3223 (Print) 0006-3223 (Linking). DOI: 10 . 1016/0006-3223 (95) 00472-6. URL: https : / www .ncbi.nlm.nih.gov/pubmed/ 8704071.

Spielberger, Charles D (1971). "Notes and comments trait-state anxiety and motor behavior". In: Journal of motor behavior 3.3, pp. 265-279. ISSN: 0022-2895.

Stanik-Hutt, Julie et al. (2013). "The Quality and Effectiveness of Care Provided by Nurse Practitioners". In: The Journal for Nurse Practitioners 9.8, 492-500.e13. ISSN: 15554155. DOI: 10.1016/j . nurpra.2013.07.004. URL: https://doi.org/10.1016/j.nurpra.2013.07. 004.

Straube, T. et al. (2009). "Sex differences in brain activation to anticipated and experienced pain in the medial prefrontal cortex". In: Hum Brain Mapp 30.2, pp. 689-98. ISSN: 10970193 (Electronic) 1065-9471 (Linking). DOI: 10 . 1002 / hbm . 20536. URL: https : / / www . ncbi.nlm.nih.gov/pubmed/18219622.

Stubbs, B. et al. (2016). "Is pain sensitivity altered in people with Alzheimer's disease? A systematic review and meta-analysis of experimental pain research". In: Exp Gerontol 82, pp. 30-8. ISSN: 1873-6815 (Electronic) 0531-5565 (Linking). DOI: 10 . 1016/j . exger . 2016. 05.016. URL: https://www.ncbi.nlm.nih.gov/pubmed/27262688.

Tekin, S. et al. (2001). “Orbitofrontal and anterior cingulate cortex neurofibrillary tangle burden is associated with agitation in Alzheimer disease". In: Ann Neurol 49.3, pp. 35561. ISSN: 0364-5134 (Print) 0364-5134 (Linking). URL: https : / www . ncbi .nlm.nih . gov/ pubmed/11261510.

Tierney, M. C. et al. (2014). "Factors associated with primary care physicians' recognition of cognitive impairment in their older patients". In: Alzheimer Dis Assoc Disord 28.4, pp. 320-5. ISSN: 1546-4156 (Electronic) 0893-0341 (Linking). DOI: 10 . 1097 / WAD . 0000000000000039 . URL: https://www.ncbi.nlm.nih.gov/pubmed/24632991.

Tolea, M. I. and J. E. Galvin (2013). "Current guidelines for dementia screening: shortcomings and recommended changes". In: Neurodegener Dis Manag 3.6, pp. 565-573. ISSN: 1758-2032 (Electronic) 1758-2024 (Linking). DOI: 10 . 2217 / nmt . 13 . 58. URL: https : //www.ncbi.nlm.nih.gov/pubmed/25152772.

Tonacci, A. et al. (2017). “Olfactory evaluation in Mild Cognitive Impairment: correlation with neurocognitive performance and endothelial function". In: Eur J Neurosci 45.10, pp. 1279-1288. ISSN: 1460-9568 (Electronic) 0953-816X (Linking). DOI: 10 . 1111 / ejn . 13565. URL: https://www.ncbi.nlm.nih.gov/pubmed/28370677.

$\mathrm{Tu}$, S. et al. (2015). "Lost in spatial translation - A novel tool to objectively assess spatial disorientation in Alzheimer's disease and frontotemporal dementia". In: Cortex 67, pp. 83- 
94. ISSN: 1973-8102 (Electronic) 0010-9452 (Linking). DOI: 10 . 1016/ j . cortex . 2015 . 03. 016. URL: https://www.ncbi.nlm.nih.gov/pubmed/25913063.

Tuwaig, M. et al. (2017). "Deficit in Central Auditory Processing as a Biomarker of PreClinical Alzheimer's Disease". In: J Alzheimers Dis 60.4, pp. 1589-1600. ISSN: 1875-8908 (Electronic) 1387-2877 (Linking). DOI: 10 .3233/JAD-170545. URL: https : / www . ncbi . nlm.nih.gov/pubmed/28984583.

Veneziani, F. et al. (2016). "Examination of level of knowledge in Italian general practitioners attending an education session on diagnosis and management of the early stage of Alzheimer's disease: pass or fail?" In: Int Psychogeriatr 28.7, pp. 1111-24. ISSN: 1741203X (Electronic) 1041-6102 (Linking). DOI: 10 . 1017 /S1041610216000041. URL: https : //www.ncbi.nlm.nih.gov/pubmed/26817511.

Verghese, P. B., J. M. Castellano, and D. M. Holtzman (2011). “Apolipoprotein E in Alzheimer's disease and other neurological disorders". In: Lancet Neurol 10.3, pp. 24152. ISSN: 1474-4465 (Electronic) 1474-4422 (Print) 1474-4422 (Linking).

Wager, T. D. et al. (2004). "Placebo-induced changes in FMRI in the anticipation and experience of pain". In: Science 303.5661, pp. 1162-7. ISSN: 1095-9203 (Electronic) 0036-8075 (Linking). DOI: 10 .1126/science.1093065. URL: https : //www . ncbi . nlm . nih . gov/ pubmed/14976306.

Waldton, S. (1974). "Clinical observations of impaired cranial nerve function in senile dementia". In: Acta Psychiatr Scand 50.5, pp. 539-47. ISSN: 0001-690X (Print) 0001-690X (Linking). DOI: 10 .1111/ j . $1600-0447$. 1974 . tb09714 . x. URL: https : / / www . ncbi . nlm.nih.gov/pubmed/4460686.

Wang, Q. S. et al. (2002). "Olfactory identification and apolipoprotein E epsilon 4 allele in mild cognitive impairment". In: Brain Res 951.1, pp. 77-81. ISSN: 0006-8993 (Print) 00068993 (Linking). DOI: 10 . 1016/s0006-8993(02) 03137-2. URL: https : / www . ncbi .nlm. nih.gov/pubmed/12231459.

Ward, A. et al. (2012). "Prevalence of apolipoprotein E4 genotype and homozygotes (APOE e4/4) among patients diagnosed with Alzheimer's disease: a systematic review and meta-analysis". In: Neuroepidemiology 38.1, pp. 1-17. ISSN: 1423-0208 (Electronic) 02515350 (Linking). DOI: 10.1159/000334607.

Whittemore, R. and K. Knafl (2005). "The integrative review: updated methodology". In: J Adv Nurs 52.5, pp. 546-53. ISSN: 0309-2402 (Print) 0309-2402 (Linking). DOI: 10.1111/j . 1365-2648.2005.03621.x. URL: https://www.ncbi.nlm.nih.gov/pubmed/16268861.

Wilkinson, D. et al. (2004). "The role of general practitioners in the diagnosis and treatment of Alzheimer's disease: a multinational survey". In: J Int Med Res 32.2, pp. 149-59. ISSN: 0300-0605 (Print) 0300-0605 (Linking). DOI: 10.1177/147323000403200207. URL: https : //www.ncbi.nlm.nih.gov/pubmed/15080018.

Wilson, R. S. et al. (2012). "The natural history of cognitive decline in Alzheimer's disease". In: Psychol Aging 27.4, pp. 1008-17. ISSN: 1939-1498 (Electronic) 0882-7974 (Linking). DOI: 10.1037/a0029857. URL: https://www.ncbi.nlm.nih.gov/pubmed/22946521. 
Yamazaki, Y. et al. (2019). "Apolipoprotein E and Alzheimer disease: pathobiology and targeting strategies". In: Nat Rev Neurol 15.9, pp. 501-518. ISSN: 1759-4766 (Electronic) 1759-4758 (Linking). DOI: 10.1038/s41582-019-0228-7. URL: https : //www . ncbi.nlm . nih.gov/pubmed/31367008.

Zissimopoulos, J., E. Crimmins, and P. St Clair (2014). “The Value of Delaying Alzheimer's Disease Onset". In: Forum Health Econ Policy 18.1, pp. 25-39. ISSN: 2194-6191 (Print) 15589544 (Linking). DOI: 10.1515/fhep-2014-0013. URL: https : / www .ncbi .nlm.nih.gov/ pubmed/27134606. 


\section{Vita}

Raymond R. Romano, III born in 1989 was raised in Kearny, New Jersey. His research is focused on the early detection and diagnosis of dementia in the primary care setting. His interest in dementia began during his undergraduate career at Marymount Manhattan College, when he studied the role a protein, Cyclin-Dependent Kinase 5 (CDK5), in insulin related neurodegenerative diseases. He explored the molecular pathway hyperinsulinemia has on the aberrant behavior of CDK5 contributing to the pathology of Alzheimer's disease. In 2011 he received his bachelor's degree in Biology from Marymount Manhattan College and went to study for a master's in public health from Boston University during which he began working in clinical research at the Boston University Alzheimer's Disease Center. After graduating in 2013 he moved to Nashville to join the Vanderbilt Memory and Alzheimer's Center. He completed the Vanderbilt University School of Nursing Family Nurse Practitioner (FNP) program as a pre-specialty student in 2015 and is currently working as a Family Nurse Practitioner in primary care practice with the Metro Nashville Public School system. Raymond began his doctoral studies at The University of Tennessee Health Science Center in Nursing Science in 2019 under the mentorship of Drs. Michael Carter, Todd Monroe, Ronald Cowan, Margaret Thorman Hartig, and Carolyn Gaff. He has published worked focused on vascular health and Alzheimer's disease pathology, subjective memory complaints, barriers of minority groups to participation in research, barriers to diagnosis of $\mathrm{AD}$ in primary care, difference in the experience of pain between the sexes across the cognitive spectrum, and difference in the experience of pain between those at varying levels of risk for AD based on carrying the $\varepsilon$ allele of apolipoprotein e. 